TRANSACTIONS OF THE

AMERICAN MATHEMATICAL SOCIETY

Volume 350, Number 11, November 1998, Pages 4669-4691

S 0002-9947(98)02029-7

\title{
A CLASS OF PARABOLIC $k$-SUBGROUPS ASSOCIATED WITH SYMMETRIC $k$-VARIETIES
}

\author{
A. G. HELMINCK AND G. F. HELMINCK
}

\begin{abstract}
Let $G$ be a connected reductive algebraic group defined over a field $k$ of characteristic not $2, \sigma$ an involution of $G$ defined over $k, H$ a $k$ open subgroup of the fixed point group of $\sigma, G_{k}$ (resp. $H_{k}$ ) the set of $k$ rational points of $G$ (resp. $H$ ) and $G_{k} / H_{k}$ the corresponding symmetric $k$ variety. A representation induced from a parabolic $k$-subgroup of $G$ generically contributes to the Plancherel decomposition of $L^{2}\left(G_{k} / H_{k}\right)$ if and only if the parabolic $k$-subgroup is $\sigma$-split. So for a study of these induced representations a detailed description of the $H_{k}$-conjucagy classes of these $\sigma$-split parabolic $k$ subgroups is needed.

In this paper we give a description of these conjugacy classes for general symmetric $k$-varieties. This description can be refined to give a more detailed description in a number of cases. These results are of importance for studying representations for real and $\mathfrak{p}$-adic symmetric $k$-varieties.
\end{abstract}

\section{INTRODUCTION}

Let $G$ be a connected reductive algebraic group defined over a field $k$ of characteristic not 2 and $\sigma$ an involution of $G$ defined over $k$. If $H$ is a $k$-open subgroup of the fixed point group of $\sigma$, then $G_{k} / H_{k}$ is called a symmetric $k$-variety. Here $G_{k}$ (resp. $H_{k}$ ) denotes the set of $k$-rational points of $G$ (resp. $H$ ). In the last few decades the representation theory of these varieties has been studied extensively for a number of base fields. The case $k=\mathbb{R}$ is probably best known. Here the representation theory and Plancherel formulas for symmetric $k$-varieties (also called semisimple symmetric spaces) have been studied by many people. Best known is the work of Harish-Chandra [13], which has been extended to general real symmetric $k$-varieties by many others, including Flensted-Jensen, Oshima, Sekiguchi, Matsuki, Brylinski, Delorme, Schlichtkrull and van den Ban (see [9, 29, 28, 8, 1, 2]). More recently several attempts have been made to begin a systematic study of the representation theory of symmetric $k$-varieties over other base fields. Examples of this are work of Lusztig and his students on symmetric $k$-varieties over finite fields (see [26]) and a number of preliminary results about the representation theory of symmetric $k$-varieties over local fields (see for example [25] and [22]). In studying the representation theory of these symmetric $k$-varieties one runs quickly into various questions about their structure and geometry. This paper deals with some questions we encountered while studying induced representations for $\mathfrak{p}$-adic symmetric $k$-varieties. This concerns the following. Let $P$ be a parabolic $k$-subgroup of $G$ with Levi decomposition $P=L N$, where $N$ is the unipotent radical of $P$. One

Received by the editors December 15, 1995 and, in revised form, December 15, 1996.

1991 Mathematics Subject Classification. Primary 20G15, 20G20, 22E15, 22E46, 53C35.

(C)1998 American Mathematical Society 
considers continuous irreducible representations $\rho$ of $P_{k}$ on a Hilbert space that are trivial on $N_{k}$. Let $\operatorname{Ind}_{P_{k}}^{G_{k}}(\rho)$ denote the representation of $G_{k}$ obtained by inducing $\rho$ from $P_{k}$ to $G_{k}$. In order that the induced representation $\operatorname{Ind}_{P_{k}}^{G_{k}}(\rho)$ contribute to the Plancherel decomposition of $L^{2}\left(G_{k} / H_{k}\right)$, it must have $H_{k}$-fixed distribution vectors. This can only be true for generic $\rho$ if $P$ is $\sigma$-split. Here one can expect that the "most continuous part" comes from the minimal $\sigma$-split parabolic $k$-subgroups. In the case $k=\mathbb{R}$, this is known to be true by the work of van den Ban and Delorme using ideas due to Harish-Chandra; see [1, 8, 10, 11, 12]. For a study of these $H_{k}$-fixed distribution vectors a detailed description of the $H_{k}$-conjugacy classes of these $\sigma$-split parabolic $k$-subgroups is needed. This is exactly what most of this paper is about. We will first give a description of these in the general case, and then give a more detailed description in the special case of groups with a Cartan involution (this includes the case of real semisimple symmetric spaces).

Every $\sigma$-split parabolic $k$-subgroup $P$ of $G$ can be characterized as $P=P(F)$ with $F$ a facet of $X_{*}(A) \otimes_{\mathbb{Z}} \mathbb{R}$ and $A$ a maximal $(\sigma, k)$-split torus of $G$ contained in $P$. So, modulo the action of the Weyl group of $A$, one can hope to characterize these $\sigma$-split parabolic $k$-subgroups as standard parabolic $k$-subgroups with respect to a maximal $k$-split torus, containing the maximal $(\sigma, k)$-split torus. Up to $H_{k^{-}}$ conjugacy of the maximal $(\sigma, k)$-split tori, this is seen to be the case:

Corollary 2.8. Let $\left\{A_{i} \mid i \in I\right\}$ be representatives of the $H_{k}$-conjugacy classes of maximal $(\sigma, k)$-split tori of $G$, and for each $i \in I$ let $A_{i}^{0} \supset A_{i}$ be a $\sigma$-stable maximal $k$-split torus of $G$ and $\Delta^{i}$ a $\sigma$-basis of $\Phi\left(A_{i}^{0}\right)$. If $P$ is any $\sigma$-split parabolic $k$-subgroup of $G$, then there exist $i \in I, h \in H_{k}, n \in N_{G_{k}}\left(A_{i}^{0}\right) \cap N_{G_{k}}\left(A_{i}\right)$ and a subset $\Delta_{1}$ of $\Delta^{i}$ such that $n h P h^{-1} n^{-1}=P_{\Delta_{1}}$.

This result can be even more refined for minimal $\sigma$-split parabolic $k$-subgroups. See Theorem 2.9 for details.

Another way to characterize the $H_{k}$-conjugacy classes of minimal $\sigma$-split parabolic $k$-subgroups is to look at the $H_{k}$-orbits on $G_{k} / P_{k}$ which are contained in $(H P)_{k}$ (see Theorem 3.1). Here $P$ is a fixed minimal $\sigma$-split parabolic $k$-subgroup of $G$. For $k$ a local field this corresponds exactly to the open orbits:

Theorem 3.6. Assume $k$ is a local field and let $\left\{A_{i} \mid i \in I\right\}$ be representatives of the $H_{k}$-conjugacy classes of maximal $(\sigma, k)$-split tori of $G$. There is a one to one correspondence between the open $H_{k}$-orbits on $G_{k} / P_{k}$ and $\bigcup_{i \in I} W\left(A_{i}\right) / W_{H_{k}}\left(A_{i}\right)$.

To obtain a more detailed description of the $H_{k}$-conjugacy classes of the minimal $\sigma$-split parabolic $k$-subgroups one will first need a description of the $H_{k}$-conjugacy classes of the maximal $(\sigma, k)$-split tori of $G$. Unfortunately, in general the maximal $(\sigma, k)$-split tori of $G$ are not conjugate under $H_{k}$. In fact in most cases there are infinitely many conjugacy classes. In a number of special cases, like symmetric $k$-varieties over local fields, there are only finitely many conjugacy classes, and for these one can get a more detailed characterization of these $H_{k}$-conjugacy classes of maximal $(\sigma, k)$-split tori (see $[17,18,19])$.

In the remainder of this paper we discuss some special cases for which one can get a more detailed description of the $H_{k}$-conjugacy classes of $\sigma$-split parabolic $k$ subgroups. These are symmetric $k$-varieties over local fields (see 2.11 ), symmetric $k$-varieties with anisotropic fixed point group (see 3.7) and finally the the case of "groups with a Cartan involution" (see section 4). The latter were introduced in [23] as a generalization of real reductive groups. For these groups there exists a second 
involution $\theta$ (the Cartan involution) which commutes with $\sigma$. As in the case of real groups most of the structure of these groups can be derived from the additional structure provided by this Cartan involution. In this case the $H_{k}$-conjugacy classes of $\sigma$-split parabolic $k$-subgroups can be reduced to $H_{k} \cap K_{k}$-conjugacy classes of $\sigma \theta$ stable parabolic $k$-subgroups. In section 4 we first show that every $\sigma$-split parabolic $k$-subgroup is $H_{k}$-conjugate to a $\sigma \theta$-stable parabolic $k$-subgroup (see Proposition 4.13). For the $\sigma \theta$-stable parabolic $k$-subgroups we can then reduce to $H_{k} \cap K_{k}$ conjugacy classes. In the remainder of section 4 we use this reduction to $H_{k} \cap K_{k^{-}}$ conjugacy classes of $\sigma \theta$-stable parabolic $k$-subgroups to refine the characterization of the orbits in the previous sections.

For $k=\mathbb{R}$ the $H_{k} \cap K_{k}$-conjugacy classes of $\sigma \theta$-stable parabolic $k$-subgroups occur in the study of the principal series representations associated with real symmetric $k$-varieties (see $[1,8]$ ). The results in section 4 provide an algebraization of the results for real reductive groups and also a generalization to the setting of "groups with a Cartan involution".

A brief outline of this paper follows. In section 1 we set the notation and review some basic facts from [23] and [16] about symmetric $k$-varieties, $(\sigma, k)$-split tori and $\sigma$-split parabolic $k$-subgroups. Then we prove some results which will be needed for the characterization of the $H_{k}$-conjugacy classes of $\sigma$-split parabolic $k$-subgroups of $G$. Section 2 is devoted to a characterization of the $H_{k}$-conjugacy classes of $\sigma$-split parabolic $k$-subgroups for general symmetric $k$-varieties, while in section 3 we give a characterization in terms of the open orbits. The final section deals with the case of "groups with a Cartan involution".

We would like to thank the referee for a number of helpful suggestions.

\section{Preliminaries and Recollections}

In this section we set the notations and recall a few results from [23], [16] and [17]. We use as our basic reference for reductive groups the papers of Borel and Tits [5, 6] and also the books of Humphreys [24] and Springer [34]. We shall follow their notations and terminology.

1.1. Given an algebraic group $G$, the identity component is denoted by $G^{0}$. We use $L(G)$ or $\mathfrak{g}$, the corresponding lower case German letter, for the Lie algebra of $G$. If $H$ is a subset of $G, N_{G}(H)$ (resp. $Z_{G}(H)$ ) is the normalizer (resp. centralizer) of $H$ in $G$. We write $Z(G)$ for the center of $G$. The commutator subgroup of $G$ is denoted by $D(G)$ or $[G, G]$.

An algebraic group defined over $k$ will also be called an algebraic $k$-group. For an extension $K$ of $k$, the set of $K$-rational points of $G$ is denoted by $G_{K}$ or $G(K)$.

If $G$ is a reductive $k$-group and $A$ a torus of $G$, then we denote by $X^{*}(A)$ (resp. $\left.X_{*}(A)\right)$ the group of characters of $A$ (resp. one-parameter subgroups of $A$ ) and by $\Phi(G, A)$ the set of the roots of $A$ in $G$. Let $W(G, A)=N_{G}(A) / Z_{G}(A)$ denote the Weyl group of $G$ relative to $A$. If $\alpha \in \Phi(G, A)$, then let $U_{\alpha}$ denote the unipotent subgroup of $G$ corresponding to $\alpha$. If $A$ is a maximal torus, then $U_{\alpha}$ is one-dimensional. Given a quasi-closed subset $\psi$ of $\Phi(G, A)$, the group $G_{\psi}$ (resp. $\left.G_{\psi}^{*}\right)$ is defined in $[5,3.8]$. If $G_{\psi}^{*}$ is unipotent, $\psi$ is said to be unipotent and often one writes $U_{\psi}$ for $G_{\psi}^{*}$.

Throughout the paper $G$ will denote a connected reductive algebraic $k$-group. 
1.2. Involutions of $G$. Let $G$ be a connected algebraic group, $\sigma$ an automorphism of $G$ of order two and $G_{\sigma}=\{g \in G \mid \sigma(g)=g\}$ the set of fixed points of $\sigma$. This is a subgroup of $G$, and is reductive if $G$ is reductive. If $G$ is semisimple and simply connected, then $G_{\sigma}$ is connected, but in general $G_{\sigma}$ is not necessarily connected. When $G$ and $\sigma$ are defined over $k$, the automorphism $\sigma$ will also be called a $k$ involution of $G$.

If $G$ is reductive and $H$ a $k$-open subgroup of $G_{\sigma}$, then we call the variety $G / H$ a symmetric variety and the variety $G_{k} / H_{k}$ a symmetric $k$-variety. Symmetric varieties are spherical.

Define a morphism $\tau: G \rightarrow G$ by $\tau(x)=x \sigma(x)^{-1}, \quad x \in G$. The image $\tau(G)$ is a closed $k$-subvariety of $G$, and $\tau$ induces an isomorphism of the coset space $G / G_{\sigma}$ onto $\tau(G)$. Note that $\tau(x)=\tau(y)$ if and only if $y^{-1} x \in G_{\sigma}$ and $\sigma(\tau(x))=\tau(x)^{-1}$ for $x \in G$.

1.3. If $T$ is a $\sigma$-stable torus of $G$, then we write $T_{\sigma}^{+}=\left(T \cap G_{\sigma}\right)^{0}$ and $T_{\sigma}^{-}=\{x \in$ $\left.T \mid \sigma(x)=x^{-1}\right\}^{0}$. It is easy to verify that the product map

$$
\mu: T_{\sigma}^{+} \times T_{\sigma}^{-} \rightarrow T, \mu\left(t_{1}, t_{2}\right)=t_{1} t_{2},
$$

is a separable isogeny. In particular $T=T_{\sigma}^{+} T_{\sigma}^{-}$, and $T_{\sigma}^{+} \cap T_{\sigma}^{-}$is a finite group. (In fact it is an elementary abelian 2-group.) The automorphisms of $\Phi(G, T)$ and $W(G, T)$ induced by $\sigma$ will also be denoted by $\sigma$.

Recall that a torus $A$ is called $\sigma$-split if $\sigma(a)=a^{-1}$ for every $a \in A$. To the symmetric $k$-variety $G_{k} / H_{k}$ one can associate a natural root system. To see this we consider the following tori:

Definition 1.4. A $k$-torus $A$ of $G$ is called $(\sigma, k)$-split if it is both $\sigma$-split and $k$-split.

Consider a maximal $(\sigma, k)$-split torus $A$ in $G$. In $[23,5.9]$ it was shown that $\Phi(G, A)$ is a root system and $N_{G_{k}}(A) / Z_{G_{k}}(A)$ is the Weyl group of this root system. We can also obtain this root system by restricting the root system of $G_{k}$. Namely, let $A^{0} \supset A$ be a $\sigma$-stable maximal $k$-split torus of $G$. Then $A=\left(A^{0}\right)_{\sigma}^{-}$, and $\Phi(G, A)$ can be identified with $\bar{\Phi}_{\sigma}=\left\{\alpha|A \neq 0| \alpha \in \Phi\left(G, A^{0}\right)\right\}$. One can also choose compatible orders on these root systems as follows:

1.5. Characterization of $\sigma$. For each $\sigma$-stable torus $A$, the morphism $\sigma: G \longrightarrow$ $G$ induces a natural action $\sigma$ on $\Phi(G, A)$. For a proper description of this action we need to refine the notion of linear order to this setting. Let $A^{0}$ be a $\sigma$-stable maximal $k$-split torus of $G$ and let $X=X^{*}\left(A^{0}\right), \Phi=\Phi\left(A^{0}\right)$ and $X_{0}(\sigma)=\{\chi \in$ $X \mid \sigma(\chi)=\chi\}$.

Definition 1.6. A linear order $\succ$ on $X$ is called a $\sigma$-order if it has the following property:

$$
\text { if } \chi \in X, \chi \succ 0 \text {, and } \chi \notin X_{0}(\sigma) \text {, then } \sigma(\chi) \prec 0 \text {. }
$$

By $[16, \S 2] \sigma$-orders on $(X, \Phi)$ exist. If $\pi$ is the natural projection from $X$ to $X / X_{0}(\sigma)$ and $\Phi_{0}(\sigma)=X_{0}(\sigma) \cap \Phi$, then we call $\bar{\Phi}_{\sigma}=\pi\left(\Phi-\Phi_{0}(\sigma)\right)$ the set of restricted roots of $\Phi$ relative to $\sigma$. If $\Delta$ is a basis of $\Phi$ with respect to a $\sigma$-order on $X$, then we write $\Delta_{0}(\sigma)=\Delta \cap \Phi_{0}(\sigma)$ and $\bar{\Delta}_{\sigma}=\pi\left(\Delta-\Delta_{0}(\sigma)\right)$. We will also call a basis of $\Phi$ with respect to a $\sigma$-order on $X$ a $\sigma$-basis of $(X, \Phi)$. We write $W_{0}(\sigma)$ for the Weyl group of $\Phi_{0}(\sigma)$, which we identify with a subgroup of $W\left(A^{0}\right)$. 
1.7. Characterization of $\sigma$ on a $\sigma$-basis. Let $\Delta$ be a $\sigma$-basis of $(X, \Phi)$. In [16, $\S 2]$ we have shown that the action of $\sigma$ on $\Phi$ can be decomposed as

$$
\sigma=-\mathrm{id} \cdot \sigma^{*} \cdot w_{0}(\sigma)
$$

where $w_{0}(\sigma) \in W_{0}(\sigma)$ is the longest element of $W_{0}(\sigma)$ with respect to $\Delta_{0}(\sigma)$ and $\sigma^{*} \in \operatorname{Aut}\left(X, \Phi, \Delta, \Delta_{0}(\sigma)\right)=\left\{\phi \in \operatorname{Aut}(X, \Phi) \mid \phi(\Delta)=\Delta\right.$ and $\left.\phi\left(\Delta_{0}(\sigma)\right)=\Delta_{0}(\sigma)\right\}$, $\left(\sigma^{*}\right)^{2}=\mathrm{id}$. Note that if $\Phi$ is irreducible, then $\sigma^{*}=\mathrm{id}$ or is a non-trivial diagram automorphism of $\Delta$. On $\Delta$ the action of $\sigma$ can now be described as follows:

(1.3) If $\alpha \in \Delta_{0}(\sigma)$, then $\sigma(\alpha)=\alpha$.

(1.4) If $\alpha \in \Delta-\Delta_{0}(\sigma)$, then $\sigma(\alpha)=-\sigma^{*}(\alpha)+\beta$ with $\sigma^{*}(\alpha) \in \Delta$ and $\beta \in \Phi_{0}(\sigma)$.

For more details see $[16, \S 2]$.

Note that in the case in which $A$ is a maximal $(\sigma, k)$-split torus and $A^{0} \supset A$ a $\sigma$-stable maximal $k$-split torus of $G$, then in fact $\bar{\Phi}_{\sigma}=\Phi(G, A)$ is a (not reduced) root system.

1.8. Properties of $Z_{G}(A)$. We will need several properties of the centralizer of a maximal $(\sigma, k)$-split torus. The key result in the study of these is the following result (see $[23,4.5])$.

Lemma 1.9. Let $A$ be a maximal $(\sigma, k)$-split torus of $G$. Let $C, L_{1}, L_{2}$ denote the central, anisotropic and isotropic factors of $Z_{G}(A)$ over $k$ respectively. Then the following conditions hold:

(i) $A$ is the unique maximal $(\sigma, k)$-split torus of $Z_{G}(A)$.

(ii) $L_{2} \subset H$.

(iii) If $A^{0}$ is any maximal $k$-split torus of $Z_{G}(A)$, then $A^{0}$ is $\sigma$-stable and moreover $C L_{1} \subset Z_{G}\left(A^{0}\right)$.

Using this result, we can now prove the following property of the Weyl group $W_{0}(\sigma)$ of $\Phi_{0}(\sigma)$.

Lemma 1.10. Let $A$ be a $\sigma$-stable maximal $k$-split torus of $G$ with $A^{-}$a maximal $(\sigma, k)$-split torus of $G$. For $\alpha \in \Phi(A)$ let $U_{\alpha}$ denote the corresponding unipotent subgroup of $G$. Then we have the following:

(i) $U_{\alpha} \subset H$, for all $\alpha \in \Phi_{0}(\sigma)$.

(ii) $W_{0}(\sigma)$ has representatives in $H_{k}$.

Proof. $(i)$. Write $Z_{G}\left(A^{-}\right)=C \cdot L_{1} \cdot L_{2}$ as an almost direct product of $k$-groups where $C, L_{1}, L_{2}$ denote respectively the central, anisotropic and isotropic factors of $Z_{G}\left(A^{-}\right)$over $k$. Then $U_{\alpha} \subset L_{2}$. Since we know by Lemma 1.9 that $L_{2} \subset H$, the result is clear.

(ii). For $\alpha \in \Phi(A)$ let $G^{\alpha}=Z_{G}\left((\operatorname{ker} \alpha)^{0}\right)$. This is a restricted rank one $k$ subgroup of $G$. Write $G^{\alpha}=C_{1} \cdot G_{1} \cdot G_{2}$ as an almost direct product of $k$-groups where $C_{1}, G_{1}, G_{2}$ denote respectively the central, anisotropic and isotropic factors of $G^{\alpha}$ over $k$. Then the reflection $s_{\alpha} \in W(A)$ corresponding to $\alpha$ has representatives in $G_{2}$. On the other hand, if $\alpha \in \Phi_{0}(\sigma)$, then $G^{\alpha} \subset Z_{G}\left(A^{-}\right)$. So $G_{2} \subset L_{2} \subset H$. It follows that for each $\alpha \in \Phi_{0}(\sigma)$ the reflection $s_{\alpha}$ has representatives in $H_{k}$. Since $W_{0}(\sigma)$ is generated by these reflections, the result follows. 
1.11. $\sigma$-split parabolic $k$-subgroups. Associated with the $(\sigma, k)$-split tori is a class of parabolic $k$-subgroups. This correspondence is as follows. Let $A$ be a $k$-split torus of $G, \Phi(G, A)$ the set of roots of $A$ in $G$ and $X_{*}(A)$ the set of one-parameter subgroups of $A$. By chambers and facets of $X_{*}(A) \otimes_{\mathbb{Z}} \mathbb{R}$, we mean those with respect to the hyperplanes $\operatorname{ker}(\alpha), \alpha \in \Phi(G, A)$. The parabolic $k$-subgroups of $G$ containing $A$ are in bijective correspondence with the facets of $X_{*}(A) \otimes_{\mathbb{Z}} \mathbb{R}$. Given a facet $F$, the corresponding parabolic $k$-subgroup $P(F)$ of $G$ containing $A$ is determined by

$$
\Phi(P(F), A)=\{\alpha \in \Phi(G, A) \mid\langle x, \alpha\rangle \geq 0, x \in F\} .
$$

For $\lambda \in X_{*}(A)$, let

$$
\Phi(\lambda, A)=\{\alpha \in \Phi(G, A) \mid\langle\lambda, \alpha\rangle \geq 0\} .
$$

If $F(\lambda)$ is the facet containing $\lambda$, i.e. the facet of $X_{*}(A) \otimes_{\mathbb{Z}} \mathbb{R}$ determined by the hyperplanes $\operatorname{ker}(\alpha)$ with $\alpha \in\{\alpha \in \Phi(G, A) \mid\langle\lambda, \alpha\rangle=0\}$, then $\Phi(P(F(\lambda)), A)=$ $\Phi(\lambda, A)$. For simplicity, we write $P(\lambda)$ for the parabolic $k$-subgroup $P(F(\lambda))$ of $G$ containing $A$.

If $A$ is a $(\sigma, k)$-split torus, then clearly any element $\lambda \in X_{*}(A)$ gives a $\sigma$-split parabolic $k$-subgroup $P(\lambda)$. Conversely, since a parabolic $k$-subgroup contains a $\sigma$-stable maximal $k$-split torus of $G$, any $\sigma$-split parabolic $k$-subgroup is of this form, as follows from the following result.

Lemma 1.12. Let $P$ be a $\sigma$-split parabolic k-subgroup of $G$ and $A$ a $\sigma$-stable maximal $k$-split torus of $P$. Then there exists $\lambda \in X_{*}\left(A^{-}\right)$such that $P=P(\lambda)$ and $P \cap \sigma(P)=Z_{G}(\lambda)$.

For a proof of this result, see $[23,4.6]$.

The minimal $\sigma$-split parabolic $k$-subgroups now are described by the maximal $(\sigma, k)$-split tori (see $[23,4.7])$.

Proposition 1.13. Let $P$ be a $\sigma$-split parabolic $k$-subgroup of $G$ and $A$ a $\sigma$-stable maximal $k$-split torus of $P$. Then the following conditions are equivalent:

(i) $P$ is a minimal $\sigma$-split parabolic $k$-subgroup of $G$.

(ii) $P \cap \sigma(P)$ has no proper $\sigma$-split parabolic $k$-subgroups.

(iii) $\sigma$ is trivial on the isotropic factor of $P \cap \sigma(P)$ over $k$.

(iv) $A^{-}$is a maximal $(\sigma, k)$-split torus of $G$, and $Z_{G}\left(A^{-}\right)=P \cap \sigma(P)$.

The minimal $\sigma$-split parabolic subgroups of $G$ are conjugate under $H$, as follows from the next result (see $[23,4.8]$ ).

Lemma 1.14. Let $P$ be a minimal $\sigma$-split parabolic $k$-subgroup of $G$ and $P_{0} a$ minimal parabolic $k$-subgroup of $G$ contained in $P$. Then the following conditions hold:

(i) $H^{0} P=H^{0} P_{0}$.

(ii) $H^{0} P_{0}$ is open in $G$.

Unfortunately the minimal $\sigma$-split parabolic $k$-subgroups are not necessarily conjugate under $H_{k}$. Similarly, the maximal $(\sigma, k)$-split tori may not be conjugate under $H_{k}$. The best we can do is the following result (see [23, 4.11]).

Proposition 1.15. Let $P$ be a minimal $\sigma$-split parabolic $k$-subgroup of $G$. Then the following conditions are equivalent:

(i) $g \in G_{k} \cap H P$.

(ii) $g \in G_{k}$, and $\mathrm{gPg}^{-1}$ is a $\sigma$-split parabolic k-subgroup of $G$. 
For the maximal $(\sigma, k)$-split tori we can prove a slightly stronger result (see [23, $10.3])$.

Proposition 1.16. Let $A_{1}$ and $A_{2}$ be maximal $(\sigma, k)$-split tori of $G$ and $A$ a maximal $k$-split torus of $G$ containing $A_{1}$. Then there exists $g \in\left(H^{0} Z_{G}(A)\right)_{k}$ such that $g A_{1} g^{-1}=A_{2}$.

The following example illustrates that in general, the minimal $\sigma$-split parabolic $k$-subgroups are not conjugate under $H_{k}$.

Example 1.17. Let $G=\mathrm{SL}(2), \sigma(x)={ }^{t} x^{-1}, B=$ the Borel subgroup of upper triangular matrices and $A$ the group of diagonal matrices. Then $G, B, A$ and $\sigma$ are defined over $\mathbb{Q} ; B$ is a minimal $\sigma$-split parabolic $\mathbb{Q}$-subgroup of $G$ and $A$ a maximal $(\sigma, \mathbb{Q})$-split torus of $G$, which is also maximal $\mathbb{Q}$-split. If $g=\left(\begin{array}{ll}a & b \\ c & d\end{array}\right) \in \mathrm{GL}(2)$, then $\sigma(g)=(\operatorname{det} g)^{-1}\left(\begin{array}{cc}d & -c \\ -b & a\end{array}\right)$ and

$$
g^{-1} \sigma(g)=(\operatorname{det} g)^{-2}\left(\begin{array}{cc}
b^{2}+d^{2} & -(a b+c d) \\
-(a b+c d) & a^{2}+c^{2}
\end{array}\right) .
$$

So if $g \in \operatorname{SL}(2)$, then $g^{-1} \sigma(g) \in A^{-}=A=N_{Z_{G}\left(A^{-}\right)}(A)$ if and only if $a b+c d=0$. It follows that $g$ is of the form $\left(\begin{array}{cc}u t & -v \\ u & v t\end{array}\right)$ with $u, v, t \in \mathbb{Q}$ and $u v\left(1+t^{2}\right)=\operatorname{det} g=1$. In this case we have

$$
g^{-1} \sigma(g)=\left(\begin{array}{cc}
v^{2}\left(1+t^{2}\right) & 0 \\
0 & u^{2}\left(1+t^{2}\right)
\end{array}\right) .
$$

Moreover from Proposition 1.15 it follows that $g B g^{-1}$ is a $\sigma$-split parabolic $\mathbb{Q}$ subgroup of $G$. That $g B g^{-1}$ does not need to be $H_{\mathbb{Q}^{-}}$conjugate to $B$ can be seen as follows. Let $y=h b \in H_{\mathbb{Q}} B_{\mathbb{Q}}, h \in H_{\mathbb{Q}}, b=\left(\begin{array}{cc}a & \stackrel{u}{u} \\ 0 & a^{-1}\end{array}\right) \in B_{\mathbb{Q}}$. Then $y^{-1} \sigma(y)=$ $b^{-1} \sigma(b) \in A$ implies that $y^{-1} \sigma(y)=\left(\begin{array}{cc}a^{-2} & 0 \\ 0 & a^{2}\end{array}\right)$. But from (1.5) it follows that $g^{-1} \sigma(g)$ is of this form if and only if we can choose $t \in \mathbb{Q}$ such that $1+t^{2}$ is a square of a rational number. It follows that $g B g^{-1}$ is not always conjugate to $B$ by an element of $H_{\mathbb{Q}}$. Note that in this case $G$ has in fact infinitely many $H_{\mathbb{Q}^{-}}$ conjugacy classes of minimal $\sigma$-split parabolic $k$-subgroups, and consequently also infinitely many $H_{\mathbb{Q}^{-}}$-conjugacy classes of maximal $(\sigma, \mathbb{Q})$-split tori of $G$.

Remark 1.18. These $\sigma$-split parabolic $k$-subgroups are of importance in the representation theory of these symmetric $k$-varieties. For example, in the case that $k=\mathbb{R}$, the representations induced from a parabolic $k$-subgroup $P$ contribute to the Plancherel decomposition of $L^{2}\left(G_{k} / H_{k}\right)$ if $P$ is a $\sigma$-split parabolic $k$-subgroup. The contributions to the most "continuous part" of the Plancherel decomposition come from minimal $\sigma$-split parabolic $k$-subgroups (see [1]).

1.19. Orbits of minimal parabolic $k$-subgroups on $G_{k} / H_{k}$. The orbits of the minimal parabolic $k$-subgroups on $G_{k} / H_{k}$ play a central role in the study of the symmetric $k$-varieties. They are also of importance for the study of the $\sigma$ split parabolic $k$-subgroups. In the following we give a brief description of these orbits, which can be found in [23]. Let $P$ be a minimal parabolic $k$-subgroup of $G, A$ a $\sigma$-stable maximal $k$-split torus of $P, N=N_{G}(A), Z=Z_{G}(A)$ and $W=W(A)=N_{G}(A) / Z_{G}(A)$ the corresponding Weyl group. As in [23, 6.7], set $\mathcal{V}_{k}=\left\{x \in G_{k} \mid \tau(x) \in N_{k}\right\}$. The group $Z_{k} \times H_{k}$ acts on $\mathcal{V}_{k}$ by $(x, z) y=x y z^{-1}$, $(x, z) \in Z_{k} \times H_{k}, y \in \mathcal{V}_{k}$. Let $V_{k}$ be the set of $\left(Z_{k} \times H_{k}\right)$-orbits on $\mathcal{V}_{k}$. If $v \in V_{k}$, we let $x(v) \in \mathcal{V}_{k}$ be a representative of the orbit $v$ in $\mathcal{V}_{k}$. The inclusion map $\mathcal{V}_{k} \rightarrow G_{k}$ 
induces a bijection of the set $V_{k}$ of $\left(Z_{k} \times H_{k}\right)$-orbits on $\mathcal{V}_{k}$ onto the set of $\left(P_{k} \times H_{k}\right)$ orbits on $G_{k}$ (see $\left.[23, \S 6]\right)$. The set $V_{k}$ is in general infinite. In a number of cases one can show that there are only finitely many $\left(P_{k} \times H_{k}\right)$-orbits on $G_{k}$. If $k$ is algebraically closed, the finiteness of $V_{k}$ was proved by Springer [35]. The finiteness of the orbit decomposition for $k=\mathbb{R}$ was discussed by Wolf [36], Rossmann [32] and Matsuki [27]. For general local fields this result can be found in Helminck-Wang [23]. An example showing that in most cases the set $V_{k}$ is infinite can be found in $[23,6.12]$.

1.20. We conclude this section by addressing the following questions, which arise in the study of the induced representations associated with these symmetric $k$ varieties. "When can a minimal parabolic $k$-subgroup $P$ be embedded in a proper $\sigma$-split parabolic $k$-subgroup, and when can $P$ be embedded in a minimal $\sigma$-split parabolic $k$-subgroup?" The latter question will prove to be related to the open orbits in $P_{k} \backslash G_{k} / H_{k}$ (see also Theorem 3.6). This all follows from the following results.

Proposition 1.21. Let $P_{0}$ be a minimal parabolic $k$-subgroup of $G$ and let $\sigma$ be as above. Then the following are equivalent.

(i) There exists a proper $\sigma$-split parabolic k-subgroup $P$ of $G$ with $P \supset P_{0}$.

(ii) $P_{0}$ is not $\sigma$-stable.

(iii) $P_{0} H$ is not closed in $G$.

Proof. $(i) \Rightarrow(i i)$. Assume $P \supset P_{0}$ a proper $\sigma$-split parabolic $k$-subgroup of $G$. Let $A \subset P_{0}$ be a $\sigma$-stable maximal $k$-split torus of $G$, which exists by $[23,2.5]$. By Lemma 1.12, there exists $\lambda \in X_{*}\left(A^{-}\right)$, such that $P=P(\lambda)$. Since $P=P(\lambda)$ is a proper $\sigma$-split parabolic $k$-subgroup of $G$, there exists $\alpha \in \Phi(A)$ such that $\langle\alpha, \lambda\rangle>0$. If $P_{0}$ were $\sigma$-stable, then by $[23,3.5] Z_{G}(A)=Z_{G}\left(A^{+}\right)$, and $A^{+}$is a maximal $k$-split torus of $H$. So also $\langle\sigma(\alpha), \lambda\rangle>0$. But since $P$ is $\sigma$-split it follows that $P$ and $\sigma(P)$ are opposite, and hence $\langle\sigma(\alpha), \lambda\rangle<0$. It follows that $P_{0}$ is not $\sigma$-stable.

(ii) $\Rightarrow($ iii $)$. If $P_{0} H$ is closed in $G$, then by $[23,1.7] P_{0} \cap \sigma\left(P_{0}\right)$ contains a parabolic $k$-subgroup of $G$ and, since $P_{0}$ is minimal, it follows that $\sigma\left(P_{0}\right)=P_{0}$, which contradicts the fact that $P_{0}$ is not $\sigma$-stable.

$($ iii $) \Rightarrow($ i $)$. Let $A \subset P_{0}$ be a $\sigma$-stable maximal $k$-split torus of $G$, and take $\lambda \in X_{*}\left(A^{-}\right)$regular. Then, since $\sigma(\lambda)=-\lambda$, the parabolic $k$-subgroup $P(\lambda)$ is $\sigma$-split. If $P(\lambda)=G$, then $\langle\alpha, \lambda\rangle=0$ for all $\alpha \in \Phi(A)$. Since $\lambda \in X_{*}\left(A^{-}\right)$is regular, it follows that $A^{+}$is a maximal $k$-split torus of $H$ and $Z_{G}(A)=Z_{G}\left(A^{+}\right)$. From $[23,3.5]$ it follows then that $P_{0}$ is $\sigma$-stable. But then by $[23,1.7] P_{0} H$ is closed in $G$, which contradicts the assumption.

Corollary 1.22. Let $P_{0}$ be a minimal parabolic k-subgroup of $G$ and let $\sigma$ be as above. Then $P_{0}$ is contained in a minimal $\sigma$-split parabolic k-subgroup $P$ of $G$ if and only if $H P$ is open in $G$.

Proof. This result follows from Proposition 1.21 and [23, 9.2].

\section{2. $H_{k}$-CONJUGACY ClASSES OF $\sigma$-SPLiT PARABOLIC $k$-SUBGROUPS}

In this section we will give a characterization of the $H_{k}$-conjugacy classes of $\sigma$-split parabolic $k$-subgroups. These conjugacy classes play a central role in the study of $H_{k}$-invariant distribution vectors of representations associated with these 
symmetric $k$-varieties $G_{k} / H_{k}$, both when $k=\mathbb{R}$ (see [13]) and when $k=\mathbb{Q}_{\mathfrak{p}}$ (see $[22])$.

2.1. Let $P$ be a minimal $\sigma$-split parabolic $k$-subgroup and let $P_{1} \subset P$ be a minimal parabolic $k$-subgroup. By [23, 2.4] there exists a $\sigma$-stable maximal $k$-split torus $A$ in $P_{1}$. The following result will be useful in the characterization of the $H_{k}$-conjugacy classes of $\sigma$-split parabolic $k$-subgroups.

Lemma 2.2. Let $A_{1}$ and $A_{2}$ be maximal $(\sigma, k)$-split tori of $G$ and $A_{i}^{0} \supset A_{i}$ maximal $k$-split tori of $G(i=1,2)$. Then $A_{1}$ and $A_{2}$ are $H_{k}$-conjugate if and only if $A_{1}^{0}$ and $A_{2}^{0}$ are $H_{k}$-conjugate.

Proof. Assume first that $h \in H_{k}$ is such that $h A_{1} h^{-1}=A_{2}$. Let $A_{3}^{0}=h A_{1}^{0} h^{-1}$. Then $A_{2}^{0}$ and $A_{3}^{0}$ are maximal $k$-split tori of $Z_{G}\left(A_{2}\right)$. Since $\left(A_{2}^{0}\right)^{+}$and $\left(A_{3}^{0}\right)^{+}$are maximal $k$-split tori of $Z_{G}\left(A_{2}\right) \cap H$, there exists $h_{1} \in\left(Z_{G}\left(A_{2}\right) \cap H\right)_{k}$ such that $h_{1}\left(A_{3}^{0}\right)^{+} h_{1}^{-1}=\left(A_{2}^{0}\right)^{+}$. But then $h_{1} h A_{1}^{0} h^{-1} h_{1}^{-1}=A_{2}^{0}$, which proves the result.

Let $\left\{A_{i} \mid i \in I\right\}$ be representatives of the $H_{k}$-conjugacy classes of maximal $(\sigma, k)$ split tori of $G$. Every $\sigma$-split parabolic $k$-subgroup is conjugate with one containing one of the $A_{i}$.

Lemma 2.3. Any $\sigma$-split parabolic k-subgroup of $G$ is conjugate under $H_{k}$ to a $\sigma$-split parabolic $k$-subgroup containing one of the $A_{i}(i \in I)$.

Proof. Assume $P$ is a $\sigma$-split parabolic $k$-subgroup of $G$. Let $P_{1} \subset P$ be a minimal $\sigma$-split parabolic $k$-subgroup and let $A \subset P_{1}$ be a $\sigma$-stable maximal $k$-split torus of $G$, which exists by $[23,2.2]$. Then by Proposition $1.13 A^{-}$is a maximal $(\sigma, k)$ split torus of $G$. Let $h \in H_{k}$ be such that $h A^{-} h^{-1}=A_{i}$, for some $i \in I$. Then $A_{i} \subset h P h^{-1}$, which proves the result.

Let $\left\{A_{i} \mid i \in I\right\}$ be as above, and for each $i \in I$ let $A_{i}^{0}$ be a maximal $k$-split torus containing $A_{i}$.

Lemma 2.4. Let $A_{i}$ be a maximal $(\sigma, k)$-split torus of $G$ and $P \supset A_{i}$ a $\sigma$-split parabolic k-subgroup. If $A_{i}^{0} \supset A_{i}$ is a maximal k-split torus of $G$, then $A_{i}^{0} \subset P$.

Proof. Let $P_{1} \supset A_{i}$ be a minimal $\sigma$-split parabolic $k$-subgroup of $G$ contained in $P$. From Proposition 1.13 it follows that $Z_{G}\left(A_{i}\right)=P_{1} \cap \sigma\left(P_{1}\right) \subset P \cap \sigma(P) \subset P$. So clearly $A_{i}^{0} \subset Z_{G}\left(A_{i}\right) \subset P$.

Lemma 2.5. Let $A$ be a maximal $(\sigma, k)$-split torus of $G, A_{1}, A_{2}$ maximal $k$-split tori of $Z_{G}(A)$ and $P_{1}, P_{2}$ minimal parabolic $k$-subgroups of $Z_{G}(A)$. Then we have the following.

(i) $A_{1}$ and $A_{2}$ are conjugate under $\left(Z_{G}(A) \cap H\right)_{k}$.

(ii) $P_{1}$ and $P_{2}$ are conjugate under $\left(Z_{G}(A) \cap H\right)_{k}$.

Proof. Write $Z_{G}(A)=C \cdot L_{1} \cdot L_{2}$ as an almost direct product of $k$-groups where $C$, $L_{1}, L_{2}$ denote respectively the central, anisotropic and isotropic factors of $Z_{G}(A)$ over $k$. Then $A_{1}$ and $A_{2}$ are maximal $k$-split tori of $C \cdot L_{2}$. Since $L_{2} \subset H$ (see [23, 4.5 (ii)]) the result is clear.

As for $(i i)$, note that we can write $P_{1}=C \cdot L_{1} \cdot \tilde{P}_{1}, P_{2}=C \cdot L_{1} \cdot \tilde{P}_{2}$ with $\tilde{P}_{1}, \tilde{P}_{2}$ minimal parabolic $k$-subgroups of $L_{2}$. Now the result follows from the fact that $L_{2} \subset H$. 
In the following we will use the notion of "standard parabolic" as in [5]. In particular if $\Delta^{0} \subset \Phi\left(A_{i}^{0}, G\right)$ is a fundamental basis and $\mathfrak{C}\left(A_{i}^{0}\right)$ the corresponding chamber, then the standard parabolic $k$-subgroups of $G$ are those $P(F)$ with $F$ a facet of $\mathfrak{C}\left(A_{i}^{0}\right)$. Note that, since the facets of $\mathfrak{C}\left(A_{i}^{0}\right)$ correspond with subsets of $\Delta^{0}$, the standard parabolic $k$-subgroups can also be described by using subsets of $\Delta^{0}$. For a facet $F$ let $\Delta^{0}(F)=\left\{\alpha \in \Delta^{0} \mid \alpha(F)=0\right\}$ be the corresponding subset of $\Delta^{0}$. Conversely if $\Delta_{1} \subset \Delta^{0}$, then we denote the corresponding facet of $\mathfrak{C}\left(A_{i}^{0}\right)$ by $F_{\Delta_{1}}$, and we will also write $P_{\Delta_{1}}$ for the standard parabolic $k$-subgroup $P\left(F_{\Delta_{1}}\right)$.

Proposition 2.6. Let $A_{i}$ be a maximal $(\sigma, k)$-split torus of $G, A_{i}^{0} \supset A_{i}$ a maximal $k$-split torus of $G, \Delta$ a $\sigma$-basis of $\Phi\left(A_{i}^{0}\right), \bar{\Delta}_{\sigma}$ the corresponding basis of $\Phi\left(A_{i}\right)$ and $\Delta_{1} \subset \Delta$. Then the following are equivalent:

(i) $P_{\Delta_{1}}$ is a $\sigma$-split parabolic k-subgroup of $G$.

(ii) There exists a subset $\Pi$ of $\bar{\Delta}_{\sigma}$ such that $\Delta_{1}=\left\{\alpha \in \Delta|\alpha| A_{i}^{-} \in \Pi \cup\{0\}\right\}$.

(iii) $\Delta_{0}(\sigma)=\{\alpha \in \Delta \mid \sigma(\alpha)=\alpha\} \subset \Delta_{1}$, and $\Phi\left(\Delta_{1}\right)=\Phi\left(A_{i}^{0}\right) \cap \mathbb{Z} \Delta_{1}$ is $\sigma$-stable.

Proof. Write $X=X^{*}\left(A_{i}^{0}\right)$ and $\Phi=\Phi\left(A_{i}^{0}\right)$.

$(i) \Rightarrow($ iii $)$. Assume $P_{\Delta_{1}}$ is $\sigma$-split. By Lemma 1.12 there exists $\lambda \in X_{*}\left(A_{i}\right)$ such that $P=P(\lambda)$ and $P \cap \theta(P)=Z_{G}(\lambda)$. Moreover, $\Delta_{1}=\{\alpha \in \Delta \mid\langle\alpha, \lambda\rangle=0\}$. Since $\lambda \in X_{*}\left(A_{i}\right)$, we have

$$
\langle\alpha, \lambda\rangle=\langle\sigma(\alpha), \sigma(\lambda)\rangle=\langle\sigma(\alpha),-\lambda\rangle .
$$

So clearly $\Delta_{0}(\sigma) \subset \Delta_{1}$. It remains to show that $\Phi\left(\Delta_{1}\right)$ is $\sigma$-stable.

As in (1.2) write $\sigma=-\mathrm{id} \sigma^{*} w_{0}(\sigma)$, where $w_{0}(\sigma) \in W_{0}(\sigma)$ is the longest element of $W_{0}(\sigma)$ with respect to $\Delta_{0}(\sigma)$ and $\sigma^{*} \in \operatorname{Aut}\left(\Phi, \Delta, \Delta_{0}(\sigma)\right),\left(\sigma^{*}\right)^{2}=$ id. If $\alpha \in$ $\Delta_{1}-\Delta_{0}(\sigma)$, then by (1.4) we have $\sigma(\alpha)=-\sigma^{*}(\alpha)+\beta$ with $\sigma^{*}(\alpha) \in \Delta-\Delta_{0}(\sigma)$ and $\beta \in \Phi_{0}(\sigma)$. It suffices to show now that $\sigma^{*}(\alpha) \in \Delta_{1}$. But since $\Delta_{0}(\sigma) \subset \Delta_{1}$ we have $\langle\beta, \lambda\rangle=0$ and hence

$$
\left\langle\sigma^{*}(\alpha), \lambda\right\rangle=\langle-\sigma(\alpha)+\beta, \lambda\rangle=-\langle\sigma(\alpha), \lambda\rangle+\langle\beta, \lambda\rangle=0+0=0 .
$$

It follows that $\sigma^{*}(\alpha) \in \Delta_{1}-\Delta_{0}(\sigma)$ and $\Phi\left(\Delta_{1}\right)$ is $\sigma$-stable.

(iii) $\Rightarrow$ (ii). Let $\Pi=\left\{\alpha\left|A_{i}\right| \alpha \in \Delta_{1}-\Delta_{0}(\sigma)\right\}$. Since $\Delta$ is a $\sigma$-basis we have $\Pi \subset \bar{\Delta}_{\sigma}$. So clearly $\Delta_{1}=\left\{\alpha \in \Delta|\alpha| A_{i}^{-} \in \Pi \cup\{0\}\right\}$.

$($ ii $) \Rightarrow($ iii $)$. Since $\sigma(\Pi)=-\Pi$, it follows that $\Phi\left(\Delta_{1}\right)$ is $\sigma$-stable and $\Delta_{0}(\sigma) \subset \Delta_{1}$.

(iii) $\Rightarrow(i)$. Let $\lambda \in X_{*}\left(A_{i}^{0}\right)$ be such that $P_{\Delta_{1}}=P(\lambda)$. Then $\Phi\left(\Delta_{1}\right)=\Phi(\lambda)$ and $\Phi\left(A_{i}^{0}, P_{\Delta_{1}}\right)=\{\alpha \in \Phi \mid\langle\alpha, \lambda\rangle \geq 0\}$. Let $\alpha \in \Delta$ with $\langle\alpha, \lambda\rangle>0$. Then $\alpha \in \Delta-\Delta_{1}$. Since $\Delta_{0}(\sigma) \subset \Delta_{1}$ we also have $\alpha \in \Delta-\Delta_{0}(\sigma)$. Similarly as above we get $\sigma(\alpha)=-\sigma^{*}(\alpha)+\beta$ with $\sigma^{*}(\alpha) \in \Delta-\Delta_{0}(\sigma)$ and $\beta \in \Phi_{0}(\sigma)$. Since $\Phi\left(\Delta_{1}\right)$ is $\sigma$-stable it follows that $\sigma^{*}(\alpha) \in \Delta-\Delta_{1}$. But then

$$
\langle\sigma(\alpha), \lambda\rangle=\left\langle-\sigma^{*}(\alpha)+\beta, \lambda\right\rangle=-\left\langle\sigma^{*}(\alpha), \lambda\right\rangle+\langle\beta, \lambda\rangle=-\left\langle\sigma^{*}(\alpha), \lambda\right\rangle<0
$$

It follows that $P_{\Delta_{1}} \cap \sigma\left(P_{\Delta_{1}}\right)=Z_{G}(\lambda)$, and hence $P_{\Delta_{1}}$ is $\sigma$-split.

Every $\sigma$-split parabolic $k$-subgroup of $G$ containing one of the $A_{i}(i \in I)$ is conjugate under $N_{G_{k}}\left(A_{i}^{0}\right) \cap N_{G_{k}}\left(A_{i}\right)$ with a standard $\sigma$-split parabolic $k$-subgroup, as follows from the following result.

Proposition 2.7. Let $P$ be a $\sigma$-split parabolic $k$-subgroup of $G$ with $P \supset A_{i}$ for some $i \in I, A_{i}^{0} \supset A_{i}$ a $\sigma$-stable maximal $k$-split torus of $G$ and $\Delta$ a $\sigma$-basis of $\Phi\left(A_{i}^{0}\right)$. Then there exists $n \in N_{G_{k}}\left(A_{i}^{0}\right) \cap N_{G_{k}}\left(A_{i}\right)$ such that $n P n^{-1}$ is a standard $\sigma$-split parabolic $k$-subgroup. 
Proof. Write $X=X^{*}\left(A_{i}^{0}\right)$ and $\Phi=\Phi\left(A_{i}^{0}\right)$. Note first that it follows from Lemma 2.4 that $A_{i}^{0} \subset P$. Let $\lambda \in X_{*}\left(A_{i}\right)$ be such that $P=P(\lambda)$ and $P \cap \sigma(P)=Z_{G}(\lambda)$. Let $\Phi(\lambda)=\{\alpha \in \Phi \mid\langle\alpha, \lambda\rangle=0\}$ be the root system of $Z_{G}(\lambda)$ with respect to $A_{i}^{0}$. Since $\sigma(\lambda)=-\lambda$ it follows that $\Phi(\lambda)$ is $\sigma$-stable.

Let $X(\lambda)=\{\chi \in X \mid\langle\chi, \lambda\rangle=0\}$. Then $X(\lambda)$ is a $\sigma$-stable cotorsion free submodule of $X$. Let $\succ$ be a $\sigma$-order on $(X(\lambda), \Phi(\lambda))$ and extend this to an order on $(X, \Phi)$ by choosing an order on $X / X(\lambda)$. Since $X(\lambda) \supset X_{0}(\sigma)$ and $X(\lambda)$ is $\sigma$ stable, it follows that $\succ$ is a $\sigma$-order on $(X, \Phi)$. Let $\Delta_{1}$ be the basis corresponding to $\succ$ and let $\Delta_{1}(\lambda)=\Delta_{1} \cap \Phi(\lambda)$ be the corresponding basis of $\Phi(\lambda)$. Note that $P=P(\lambda)=P_{\Delta(\lambda)}$.

Since $\Delta$ and $\Delta_{1}$ are both $\sigma$-bases of $\Phi$, they induce bases $\bar{\Delta}_{\sigma}$ and $\overline{\left(\Delta_{1}\right)_{\sigma}}$ of $\bar{\Phi}_{\sigma}=\Phi\left(A_{i}\right)$. Since $A_{i}$ is a maximal $(\sigma, k)$-split torus of $G$ and $\Phi\left(A_{i}\right)$ is a root system, there exists $w \in W\left(A_{i}\right)$ such that $w\left(\overline{\left(\Delta_{1}\right)_{\sigma}}\right)=\bar{\Delta}_{\sigma}$. Then $w\left(\Delta_{1}\right)$ and $\Delta$ are $\sigma$-bases of $\Phi$ which induce the same restricted basis of $\Phi\left(A_{i}\right)$. It follows now from $[16,2.5]$ that there exists $w_{0} \in W_{0}(\sigma)$ such that $w_{0} w\left(\Delta_{1}\right)=\Delta$. Let $n, n_{0} \in N_{G_{k}}\left(A_{i}^{0}\right) \cap N_{G_{k}}\left(A_{i}\right)$ be representatives of $w$ and $w_{0}$ respectively. If $\Delta_{2}=$ $w_{0} w(\Delta(\lambda)) \subset \Delta$, then $P_{\Delta_{2}}=n_{0} n P n^{-1} n_{0}^{-1}$ is a standard parabolic $k$-subgroup of $G$. Since $w_{0} w \sigma=\sigma w_{0} w$, it follows that $\Phi\left(\Delta_{2}\right)=w_{0} w \Phi(\lambda)$ is $\sigma$-stable. But since clearly $\Delta_{0}(\sigma) \subset \Delta_{2}$, it follows now from Proposition 2.6 that $\Phi\left(\Delta_{2}\right)$ is $\sigma$-split.

Combining this with Lemma 2.3, we now get the following result:

Corollary 2.8. Let $\left\{A_{i} \mid i \in I\right\}$ be representatives of the $H_{k}$-conjugacy classes of maximal $(\sigma, k)$-split tori of $G$, and for each $i \in I$ let $A_{i}^{0} \supset A_{i}$ be a $\sigma$-stable maximal $k$-split torus of $G$ and $\Delta^{i}$ a $\sigma$-basis of $\Phi\left(A_{i}^{0}\right)$. If $P$ is any $\sigma$-split parabolic $k$-subgroup of $G$, then for $i \in I$ there exist $h \in H_{k}, n \in N_{G_{k}}\left(A_{i}^{0}\right) \cap N_{G_{k}}\left(A_{i}\right)$ and a subset $\Delta_{1}$ of $\Delta^{i}$ such that $n h P h^{-1} n^{-1}=P_{\Delta_{1}}$.

Proof. This result is immediate from Lemma 2.3, Proposition 2.6 and Proposition 2.7 .

For the minimal $\sigma$-split parabolic $k$-subgroups of $G$ we can even show a more detailed characterization. If we denote the set of minimal $\sigma$-split parabolic $k$ subgroups of $G$ containing a maximal $(\sigma, k)$-split torus $A$ by $\mathfrak{P}(A)$, then we have the following result:

Theorem 2.9. For each $i \in I$, let $A_{i}$ be a maximal $(\sigma, k)$-split torus of $G, A_{i}^{0} \supset A_{i}$ a $\sigma$-stable maximal $k$-split torus of $G, \Delta^{i}$ a $\sigma$-basis of $\Phi\left(A_{i}^{0}\right)$ and $\Delta_{0}^{i}(\sigma)=\{\alpha \in$ $\left.\Delta^{i} \mid \sigma(\alpha)=\alpha\right\}$. Then we have the following.

(i) For each $i \in I, P_{\Delta_{0}^{i}(\sigma)}$ is a minimal $\sigma$-split parabolic $k$-subgroup of $G$.

(ii) If $P$ is any minimal $\sigma$-split parabolic $k$-subgroup of $G$, then for $i \in I$ there exist $h \in H_{k}$ and $n \in N_{G_{k}}\left(A_{i}^{0}\right) \cap N_{G_{k}}\left(A_{i}\right)$ such that $n h P h^{-1} n^{-1}=P_{\Delta_{0}^{i}(\sigma)}$.

(iii) There is a bijective correspondence between $\mathfrak{P}\left(A_{i}\right)$ and the bases for $\Phi\left(A_{i}\right)$.

(iv) For each $i \in I$ the group $W\left(A_{i}\right)$ acts simply transitively on $\mathfrak{P}\left(A_{i}\right)$.

(v) There is a bijective correspondence between the $H_{k}$-conjugacy classes of the minimal $\sigma$-split parabolic $k$-subgroups in $\mathfrak{P}\left(A_{i}\right)$ and $W\left(A_{i}\right) / W_{H_{k}}\left(A_{i}\right)$.

Proof. (i). Let $P=P_{\Delta_{0}^{i}(\sigma)}$. Since $\Phi_{0}(\sigma)$ is $\sigma$-stable it follows from Proposition 2.6 that $P$ is $\sigma$-split. By Lemma 1.12 there exists $\lambda \in X_{*}\left(A_{i}\right)$ such that $P=P(\lambda)$ and $P \cap \theta(P)=Z_{G}(\lambda)$. Let $\Phi(\lambda)=\left\{\alpha \in \Phi\left(A_{i}\right) \mid\langle\alpha, \lambda\rangle=0\right\}$. Since $\Delta_{0}^{i}(\sigma) \subset \Phi(\lambda)$, it 
follows that $\Phi(\lambda)=\Phi\left(\Delta_{0}(\sigma)\right)=\Phi_{0}(\sigma)$. But then $Z_{G}(\lambda)=Z_{G}\left(A_{i}\right)$, and hence it follows from Proposition 1.13(iv) that $P$ is minimal $\sigma$-split.

(ii). Let $P$ be a minimal $\sigma$-split parabolic $k$-subgroup of $G$. By Lemma 2.3 there exists $h \in H_{k}$ such that $h P h^{-1} \supset A_{i}$ for some $i \in I$. The result now follows from Proposition 2.7.

(iii). Since each $w \in W\left(A_{i}\right)$ has a representative in $N_{G_{k}}\left(A_{i}^{0}\right) \cap N_{G_{k}}\left(A_{i}\right)$, the group $W\left(A_{i}\right)$ acts on $\mathfrak{P}\left(A_{i}\right)$.

Let $P \in \mathfrak{P}\left(A_{i}\right)$ be a minimal $\sigma$-split parabolic $k$-subgroup of $G$. By Lemma 2.4 we have $P \supset A_{i}^{0}$, and by Proposition 2.7 there exists $n \in N_{G_{k}}\left(A_{i}^{0}\right) \cap N_{G_{k}}\left(A_{i}\right)$ be such that $n P n^{-1}$ is standard. Let $\Delta_{1} \subset \Delta^{i}$ be such that $n P n^{-1}=P_{\Delta_{1}}$. From Proposition 2.7 it follows that $\Delta_{1} \supset \Delta_{0}^{i}(\sigma)$, but since $P$ is minimal $\sigma$-split it follows from $(i)$ that $\Delta_{1}=\Delta_{0}^{i}(\sigma)$. We obtain a bijective correspondence between the bases for $\Phi\left(A_{i}\right)$ and $\mathfrak{P}\left(A_{i}\right)$.

(iv). Since $W\left(A_{i}\right)$ acts simply transitive on the bases of $\Phi\left(A_{i}\right),(i v)$ follows from (iii).

$(v)$. Assume $P_{1}, P_{2} \in \mathfrak{P}\left(A_{i}\right)$ are $H_{k}$-conjugate. Let $h \in H_{k}$ be such that $h P_{1} h^{-1}=$ $P_{2}$ and let $A=h A_{i}^{0} h^{-1}$. Then $A$ and $A_{i}^{0}$ are $\sigma$-stable maximal $k$-split tori of $P_{2}$. Since $A^{-} \subset P_{2} \cap \sigma\left(P_{2}\right)=Z_{G}\left(A_{i}\right)$, it follows that $A^{-} A_{i}$ is a $(\sigma, k)$-split torus of $G$. But since $A_{i}$ is maximal $(\sigma, k)$-split it follows that $A^{-}=A_{i}$. So $A$ and $A_{i}^{0}$ are $\sigma$-stable maximal $k$-split tori of $Z_{G}\left(A_{i}\right)$. By Lemma 2.5 there exists $h \in$ $\left(Z_{G}\left(A_{i}\right) \cap H\right)_{k}$ such that $h A h^{-1}=A_{i}^{0}$. It follows that $P_{1}$ and $P_{2}$ are conjugate under $N_{H_{k}}\left(A_{i}^{0}\right) \cap N_{H_{k}}\left(A_{i}\right)$, which proves the result.

Remark 2.10. In order to give a more detailed description, or possibly even a classification, of the $H_{k}$-conjugacy classes of minimal $\sigma$-split parabolic $k$-subgroups, one first needs a classification of the $H_{k}$-conjugacy classes of maximal $(\sigma, k)$-split tori of $G$. This classification follows from the classification of the $H_{k}$-conjugacy classes of $\sigma$-stable maximal $k$-split tori of $G$. An initial study of these conjugacy classes was carried out in $[17,18,19]$ (see also [20]). However a full classification of these conjugacy classes has only been completed for $k$ algebraically closed or the real numbers. In order to classify these conjugacy classes over other base fields, one will need first a classification of the symmetric $k$-varieties for those fields. Some of the necessary fine structure related to these symmetric $k$-varieties was classified in [15] (see also [21]), but a classification of the quadratic elements characterizing the isomorphy classes is still needed. Once the classification of the symmetric $k$-varieties is finished, one can try to classify the $H_{k}$-conjugacy classes of maximal $(\sigma, k)$-split tori for a number of base fields. Note that in most cases there are in fact infinitely many $H_{k}$-conjugacy classes of maximal $(\sigma, k)$-split tori, as can be seen from Example 1.17. In the case of local fields there are only finitely many conjugacy classes and a classification will be feasible. In section 4 we discuss a special case, where there is only one $H_{k}$-conjugacy class of maximal $(\sigma, k)$-split tori and for which we can give a more detailed description of the $H_{k}$-conjugacy classes of minimal $\sigma$-split parabolic $k$-subgroups. In the case of local fields we can also give a slightly more detailed description of the conjugacy classes as follows (see also Theorem 3.6).

2.11. Orbits over local fields. In the case that $k$ is a local field, we can refine some of the above results. So for the remainder of this section assume that $k$ is a local field. Let $A$ be a maximal $(\sigma, k)$-split torus of $G, A_{0} \supset A$ a maximal $k$-split torus of $G, P_{1} \supset A$ a minimal $\sigma$-split parabolic $k$-subgroup in $\mathfrak{P}(A), P \subset P_{1}$ a minimal parabolic $k$-subgroup of $G$ with $A_{0} \subset P$. By [7] there exists an " $A_{0}$-good" 
maximal compact subgroup $K_{k}$ of $G_{k}$ such that $G_{k}=K_{k} P_{k}=P_{k} K_{k}$ (Iwasawa decomposition).

We now have the following generalization of Corollary 2.8 and Proposition 2.9(ii):

Proposition 2.12. Assume $k$ is a local field, and let $\left\{A_{i} \mid i \in I\right\}$ be representatives of the $H_{k}$-conjugacy classes of maximal $(\sigma, k)$-split tori of $G$. For each $i \in I$ let $A_{i}^{0} \supset A_{i}$ be a maximal $k$-split torus of $G, \Delta^{i}$ a $\sigma$-basis of $\Phi\left(A_{i}^{0}\right), \Delta_{0}^{i}(\sigma)=\{\alpha \in$ $\left.\Delta^{i} \mid \sigma(\alpha)=\alpha\right\}$ and $K_{k}^{i}$ an $A_{i}^{0}$-good maximal compact subgroup of $G_{k}$ as in 2.11. If $P$ is any $\sigma$-split parabolic $k$-subgroup of $G$, then we have the following:

(i) There exist (for $i \in I) h \in H_{k}, n \in N_{K_{k}}\left(A_{i}^{0}\right) \cap N_{K_{k}}\left(A_{i}\right)$ and a subset $\Delta_{1}$ of $\Delta^{i}$ such that $n h P h^{-1} n^{-1}=P_{\Delta_{1}}$.

(ii) If $P$ is a minimal $\sigma$-split parabolic $k$-subgroup of $G$, then for $i \in I$ there exist $h \in H_{k}$ and $n \in N_{K_{k}}\left(A_{i}^{0}\right) \cap N_{K_{k}}\left(A_{i}\right)$ such that $n h P h^{-1} n^{-1}=P_{\Delta_{0}^{i}(\sigma)}$.

(iii) The number of $H_{k}$-conjugacy classes of minimal $\sigma$-split parabolic $k$-subgroups is finite.

Proof. By Corollary 2.8 there exist $i \in I$ and $h \in H_{k}$ such that $h P h^{-1} \supset A_{i}^{0}$. Since by [7], $W\left(A_{i}^{0}\right)$ has representatives in $K_{k}^{i},(i)$ follows from Corollary 2.8 and $(i i)$ follows from Proposition 2.9(ii).

Finally (iii) follows from the fact that for $k$ a local field there are only finitely many $H_{k}$-orbits on $G_{k} / P_{k}$ (see $[23,6.15]$ ).

\section{Characterization of $(H P)_{k}$}

Instead of $H_{k}$-conjugacy classes of minimal $\sigma$-split parabolic $k$-subgroups one could study the more general problem of $H_{k}$-orbits on $G_{k} / P_{k}$ (or equivalently $H_{k} \times$ $P_{k}$-orbits on $G_{k}$ ). Naturally not all parabolic subgroups contained in $G_{k} / P_{k}$ are still $\sigma$-split. Similar to [18], one could define these to be "quasi $\sigma$-split" (i.e. parabolic $k$ subgroups of $G$ which are $G_{k}$-isomorphic to a $\sigma$-split parabolic $k$-subgroup). Using a similar approach as in [18] and [23, §6], one can derive a characterization of these $H_{k}$-orbits on $G_{k} / P_{k}$ in terms of $H_{k}$-conjugacy classes of $\sigma$-stable quasi $(\sigma, k)$-split tori (i.e. $k$-tori which are $G_{k}$-isomorphic to a $(\sigma, k)$-split torus) and their Weyl groups. This characterization is quite technical and hard to derive. On the other hand, for the study of the principal series representations on these symmetric $k$ varieties we mainly need a characterization of the "open orbits", which is equivalent to a characterization of the $H_{k} \times P_{k}$-orbits in $(H P)_{k}$, for $P$ a minimal $\sigma$-split parabolic $k$-subgroup of $G$. The following result characterizes the orbits of $H_{k}$ on $G_{k} / P_{k}$ contained in the open orbit $H P$.

Theorem 3.1. Let $\left\{A_{i} \mid i \in I\right\}$ be representatives of the $H_{k}$-conjugacy classes of maximal $(\sigma, k)$-split tori of $G$. There is a one to one correspondence between the $H_{k} \times P_{k}$-orbits on $G_{k}$ contained in $(H P)_{k}$ and $\bigcup_{i \in I} W\left(A_{i}\right) / W_{H_{k}}\left(A_{i}\right)$. In particular the orbits are characterized by $H_{k} n P_{k}$ with $n$ a representative for $W\left(A_{i}\right) / W_{H_{k}}\left(A_{i}\right)$ in $N_{G_{k}}\left(A_{i}\right)(i \in I)$.

Proof. If $g \in(H P)_{k}$, then $g P g^{-1}$ is $\sigma$-split. Indeed, write $g=h p$ with $h \in H$ and $p \in P$. Then $g P g^{-1}=h P h^{-1}$ and consequently $g P g^{-1}$ and $\sigma\left(g P g^{-1}\right)$ are opposite, which proves that $g P g^{-1}$ is $\sigma$-split.

On the other hand if $P_{1}$ is minimal $\sigma$-split, then by [23, 4.11] there exists $g \in$ $(H P)_{k}$ such that $P_{1}=g P g^{-1}$. It follows that we have a one-to-one correspondence between $H_{k} \backslash(H P)_{k} / P_{k}$ and the $H_{k}$-conjugacy classes of minimal $\sigma$-split parabolic 
$k$-subgroups. By Lemma 2.3 every minimal $\sigma$-split parabolic $k$-subgroup of $G$ is $H_{k}$-conjugate with one containing one of the $A_{i}(i \in I)$. But by Theorem 2.9 $(v)$ the $H_{k}$-conjugacy classes of minimal $\sigma$-split parabolic $k$-subgroups in $\mathfrak{P}\left(A_{i}\right)$ correspond bijectively with $W\left(A_{i}\right) / W_{H_{k}}\left(A_{i}\right)$, which proves the result.

Another way to characterize the sets $W\left(A_{i}\right) / W_{H_{k}}\left(A_{i}\right)$ is in terms of the restricted Weyl group of $G$ as follows:

Lemma 3.2. Let $A$ a maximal $(\sigma, k)$-split torus of $G$ and $A^{0} \supset A$ be a $\sigma$-stable maximal $k$-split torus of $G$. Let $W\left(A, A^{0}\right)=W(A) \cap W\left(A^{0}\right)=\left\{w \in W\left(A^{0}\right) \mid\right.$ $w(A) \subset A\}$. Then

$$
W(A) / W_{H_{k}}(A) \simeq W\left(A, A^{0}\right) / W_{H_{k}}\left(A^{0}\right) .
$$

Proof. Let $\Phi_{0}(\sigma)=\left\{\alpha \in \Phi\left(A^{0}\right) \mid \sigma(\alpha)=\alpha\right\}$, and $W_{0}(\sigma)$ the Weyl group of $\Phi_{0}(\sigma)$, which we identify with a subgroup of $W\left(A^{0}\right)$. From [33, Proposition 2.1.4] it follows that

$$
W(A) \simeq W\left(A, A^{0}\right) / W_{0}(\sigma)
$$

Since by Lemma $1.10 W_{0}(\sigma)$ has representatives in $H_{k}$, the result follows.

The orbits which are not contained in the open orbit $H P$ can be described as follows:

Proposition 3.3. Let $P$ be a minimal $\sigma$-split parabolic k-subgroup of $G, A$ a $\sigma$ stable maximal $k$-split torus of $G$ with $A^{-}$a maximal $(\sigma, k)$-split torus of $G$, and $H_{k} g P_{k}$ an $H_{k} \times P_{k}$-orbit on $G_{k}$ which is not contained in $(H P)_{k}$. Then there exists $x \in G_{k}$ such that $H_{k} x P_{k}=H_{k} g P_{k}$ and $x A x^{-1}$ is $\sigma$-stable and

$$
\operatorname{dim}\left(x A x^{-1} \cap H\right)>\operatorname{dim}\left(A^{+}\right) .
$$

Proof. Let $P_{0} \subset P$ be a minimal parabolic $k$-subgroup with $A \subset P_{0}$. Then $g A g^{-1}$ is a maximal $k$-split torus of $g P_{0} g^{-1}$. By $[23,2.4]$ there exists $h \in\left(H \cap R_{u}\left(g P_{0} g^{-1}\right)\right)_{k}$ such that $A_{1}=h g A g^{-1} h^{-1}$ is $\sigma$-stable. Let $x=h g$. Clearly $H_{k} x P_{k}=H_{k} g P_{k}$ and $A_{1}=x A x^{-1}$ is $\sigma$-stable. So it remains to show that $\operatorname{dim} A_{1}^{+}=\operatorname{dim}\left(x A x^{-1} \cap H\right)>$ $\operatorname{dim}\left(A^{+}\right)$. If $\operatorname{dim} A_{1}^{+}=\operatorname{dim}\left(A^{+}\right)$, then $A_{1}^{-}$is a maximal $(\sigma, k)$-split torus of $G$. By Lemma 1.16 there exists $y \in\left(H Z_{G}\left(A_{1}\right)\right)_{k}$ such that $y A_{1} y^{-1}=A$. But then $H_{k} x P_{k} \subset(H P)_{k}$, which contradicts the assumption.

3.4. Open orbits over local fields. In the case that $k$ is a local field we also have the topology on $G_{k}$ induced from the field $k$, and in this case Theorem 3.1 in fact characterizes precisely the open $H_{k}$-orbits on $G_{k} / P_{k}$. So for the remainder of this section we assume that $k$ is a local field. We first recall the following characterization of the open orbits of a minimal parabolic $k$-subgroup acting on the symmetric $k$-variety $G_{k} / H_{k}$ (see $\left.[23,13.4]\right)$.

Proposition 3.5. Let $k$ be a local field, $\sigma$ an involution of $G$ defined over $k, H$ an open $k$-subgroup of $G_{\sigma}$ and $P$ a minimal parabolic k-subgroup of $G$. Assume the topology on $G_{k}$ is the one induced from that of $k$. Then the following conditions are equivalent:

(i) $P$ is contained in a minimal $\sigma$-split parabolic $k$-subgroup of $G$.

(ii) $P_{k} H_{k}$ is open in $G_{k}$.

It follows from this result and [23, 9.2] that $H_{k} g P_{k}$ is open in $G_{k}$ if and only if $H g P$ is also open in $G$. This leads to the following characterization of the open $H_{k}$-orbits on $G_{k} / P_{k}$. 
Theorem 3.6. Assume $k$ is a local field and let $\left\{A_{i} \mid i \in I\right\}$ be representatives of the $H_{k}$-conjugacy classes of maximal $(\sigma, k)$-split tori of $G$. There is a one to one correspondence between the open $H_{k}$-orbits on $G_{k} / P_{k}$ and $\bigcup_{i \in I} W\left(A_{i}\right) / W_{H_{k}}\left(A_{i}\right)$.

Proof. If $H_{k} g P_{k}$ is open in $G_{k}$, then $H g P$ is also open in $G$. This yields that $H P$ and $H g P$ are the same open orbit of $P$ in $H \backslash G$; hence $g \in(H P)_{k}$. Conversely if $H_{k} x P_{k} \subset(H P)_{k}$, then by Proposition $3.5 H_{k} x P_{k}$ is open in $G_{k}$. The result follows now from Theorem 3.1.

3.7. Anisotropic fixed point group. In the case that $H$ is anisotropic over $k$ a lot more is known about the structure and geometry of the corresponding symmetric $k$-varieties. Consequently the representation theory of these symmetric $k$-varieties is studied before one attempts the general case. For example in the case that $k=\mathbb{R}$, the corresponding symmetric $k$-varieties, also called Riemannian symmetric spaces, were studied long before the non-Riemannian symmetric spaces (see [13]). In the following we show how the above results can be refined in this special case. So for the rest of this section assume that $H$ is anisotropic over $k$. From $[23,10.5]$ it follows that all $k$-orbits are contained in the open orbit $H P$ :

Proposition 3.8. Let $\sigma$ be an involution of $G$ defined over $k$ and $H$ be an open $k$-subgroup of $G_{\sigma}$. If $[G, G] \cap H$ is anisotropic over $k$, then $G_{k}=\left(P H^{0}\right)_{k}$ for any minimal parabolic k-subgroup $P$ of $G$.

3.9. Let $A$ be a $\sigma$-stable maximal $k$-split torus of $G$. Since $H$ is anisotropic over $k$ it follows that $A$ is also $\sigma$-split. Contrary to the case that $k=\mathbb{R}$, the torus $A$ does not need to be maximal $\sigma$-split. Consequently one cannot expect that all maximal $(\sigma, k)$-split tori are conjugate under $H_{k}$. To ensure these properties we need to impose additional conditions, which are satisfied in the case that $k=\mathbb{R}$ and also for some of the $\mathfrak{p}$-adic symmetric $k$-varieties.

Definition 3.10. Let $\sigma$ be an involution of $G$ defined over $k$ and let $H$ be an open $k$-subgroup of $G_{\sigma}$. We will call the symmetric pair $(G, H)$ a $(\sigma, k)$-anisotropic pair if it satisfies the following conditions:

(1) $[G, G] \cap H$ is anisotropic over $k$.

(2) All $\sigma$-stable maximal $k$-split tori of $G$ are maximal $\sigma$-split.

(3) For any $\sigma$-stable maximal $k$-split torus $A$ of $G$ we have

$$
\left(H^{0} A\right)_{k}=H_{k}^{0} A_{k}
$$

For these $(\sigma, k)$-anisotropic pairs we can now establish the following results:

Theorem 3.11. Let $(G, H)$ be a $(\sigma, k)$-anisotropic pair, $A$ a $\sigma$-stable maximal $k$ split torus of $G$ and $P \supset A$ a minimal parabolic $k$-subgroup with unipotent radical $U=R_{u}(P)$. Then we have the following.

(i) $\left(H^{0} Z_{G}(A)\right)_{k}=\left(H^{0} A\right)_{k}=H_{k} A_{k}$.

(ii) $G_{k}=\left(H^{0} P\right)_{k}=H_{k} P_{k}$.

(iii) All $\sigma$-stable maximal $k$-split tori of $G$ are conjugate under $H_{k}$.

(iv) $N_{G_{k}}(A)=N_{H_{k}}(A) Z_{G_{k}}(A)$.

Proof. Since $A$ is maximal $\sigma$-split, $(i)$ follows from 1.9 and condition (3.1).

(ii). From [23, Lemma 10.2] it follows that

$$
\left(H^{0} P\right)_{k}=\left(H^{0} Z_{G}(A)\right)_{k} U_{k}=H_{k} A_{k} U_{k} .
$$

But then the result follows from $(i)$ and Proposition 3.8. 
(iii) is immediate from $(i i)$ and Lemma 2.5. Finally, $(i v)$ follows from $(i i),(i i i)$ and Theorem 3.1 .

Remark 3.12. Note that the condition that $(G, H)$ is a $(\sigma, k)$-anisotropic pair implies that the group $G_{k}$ has an Iwasawa type decomposition: $G_{k}=H_{k} A_{k} U_{k}$.

\section{Groups with a Cartan involution}

In a number of cases one can give a more detailed description of the $H_{k}$-conjugacy classes of $\sigma$-split parabolic $k$-subgroups. In this section we discuss one such special case, namely the "groups with a Cartan involution". This includes the case of real symmetric $k$-varieties, also called reductive symmetric spaces.

4.1. Cartan involutions. In the study of real reductive groups and their representations the Cartan involution has been one of the essential tools (see [14]). In [23] the notion of Cartan involution was extended to groups defined over formally real fields satisfying the additional condition $\left(k^{\times}\right)^{2}=\left(k^{\times}\right)^{4}$. Recall that a field is called formally real if -1 is not the sum of squares (see $[3,30]$ ). The Cartan involutions are defined as follows (see [23, 11.8]).

Definition 4.2. Let $k$ be a formally real field, $G$ a connected reductive algebraic $k$-group, $\theta$ an involution of $G$ defined over $k$ and $A$ a maximal $(\theta, k)$-split torus of $G$. Let $K=G_{\theta}$ be the fixed point group of $\theta$. We call $\theta$ a Cartan involution of $G$ (over $k$ ) if $K$ is anisotropic over $k,-1 \notin\left(k^{\times}\right)^{2}=\left(k^{\times}\right)^{4}$ and $G_{k}$ satisfies one of the following equivalent conditions:

(i) $G_{k}=K_{k}^{0} A_{k} K_{k}^{0}$.

(ii) $G_{k}=K_{k} A_{k} K_{k}$.

(iii) $G_{k}=U_{k} A_{k} K_{k}$ and $\tau\left(G_{k}\right)$ consists of $k$-split semisimple elements.

(iv) $G_{k}=U_{k} A_{k} K_{k}^{0}$ and $\tau\left(G_{k}\right)$ consists of $k$-split semisimple elements.

(v) $G_{k}=\tau\left(G_{k}\right) G_{\theta}(k)$ and $\tau\left(G_{k}\right)$ consists of $k$-split semisimple elements.

Remark 4.3. The condition $-1 \notin\left(k^{\times}\right)^{2}=\left(k^{\times}\right)^{4}$ is naturally satisfied in the case that $k$ is "real closed", i.e. $k$ is formally real, but has no formally real proper algebraic extension field (see [30, 3.2]). In fact in this case the Cartan involutions for the classical real reductive groups generalize to reductive groups over real closed fields. It is an open question if the Cartan involutions for reductive groups over real closed fields are the same as those for the real reductive groups. An example of a Cartan involution for a reductive group over a real closed field is given in the following:

Example 4.4. Let $k$ be a real closed field, $G=\mathrm{SL}_{n}(k), \theta(g)={ }^{t} g^{-1}$ and $K=$ $G_{\theta}=\left\{g \in G \mid{ }^{t} g=g^{-1}\right\}=\mathrm{SO}_{n}(k)$. The group $A$ of diagonal matrices is a maximal $(\theta, k)$-split torus of $G$, which is also a maximal $k$-torus. Now $\theta$ is a Cartan involution of $G$. For $n=2$ this can be seen as follows. The set $\tau(G)$ consist of the symmetric $k$-matrices. If $\left(\begin{array}{ll}a & b \\ c & d\end{array}\right) \in \mathrm{SL}_{2}(k)$ and $\left(\begin{array}{cc}p & q \\ -q & p\end{array}\right) \in \mathrm{SO}_{2}(k)$, then $x=\left(\begin{array}{ll}a & b \\ c & d\end{array}\right)\left(\begin{array}{cc}p & q \\ -q & p\end{array}\right)=\left(\begin{array}{ll}a p-b q & a q+b p \\ c p-d q & c q+d p\end{array}\right) \in \tau(G)$ if and only if $(a+d) q=(c-b) p$. If $a+d=0$ we can take $p=0$. If $a+d \neq 0$, then we can take $q=\frac{(c-b)}{(a+d)} p$ and $p \in k$ such that $p^{2}\left(1+\frac{(c-b)^{2}}{(a+d)^{2}}\right)=p^{2}+q^{2}=1$. So we may assume $x \in \tau(G)$. But then by $[30,2.5]$ there exists $h \in \mathrm{SO}_{2}(k)$ such that $h x^{t} h \in A$. It follows from $4.2(v)$ that $\theta$ is a Cartan involution of $G$. 
For the groups with a Cartan involution as defined above, most of their structure can be derived from the additional structure provided by the Cartan involution. A thorough discussion of the groups with a Cartan involution can be found in [23, $\S 11]$. One of the main properties of these groups which we will use, is the following (see $[23,11.17])$.

Lemma 4.5. If $\sigma \in \operatorname{Aut}(G)$ is a $k$-involution, then there exists a Cartan involution $\theta \in \operatorname{Aut}(G)$ which commutes with $\sigma$.

For the rest of this section we assume $\sigma \in \operatorname{Aut}(G)$ is a $k$-involution and $\theta \in$ $\operatorname{Aut}(G)$ is a Cartan involution satisfying $\sigma \theta=\theta \sigma$. We will use the same notation as in section 1. In particular, $H$ denotes a $k$-open subgroup of $G_{\sigma}$ and $K=G_{\theta}$.

4.6. In the description of the relevant principal series representations for reductive symmetric spaces (see $[1,8]) H_{k} \cap K_{k}$-conjugacy classes of $\sigma \theta$-stable parabolic $k$ subgroups are used, and these are characterized in a similar way as is done in the previous sections for the $\sigma$-split parabolic $k$-subgroups. In the following we first establish the correspondence between the $H_{k}$-conjugacy classes of $\sigma$-split parabolic $k$-subgroups and the $H_{k} \cap K_{k}$-conjugacy classes of $\sigma \theta$-stable parabolic $k$-subgroups, and use this to refine the characterization of the orbits in 2.9 and 3.1. We note that for $k=\mathbb{R}$ some of the results about $\sigma \theta$-stable parabolic $k$-subgroups can be found in [1]. However the proofs and arguments used here provide an algebraization of those results and hold also in the context of "groups with a Cartan involution" as defined above.

To establish the above correspondence we first need to prove a few results about the $\sigma \theta$-stable parabolic $k$-subgroups. We recall the following result from [23]:

Proposition 4.7. Let $\sigma \in \operatorname{Aut}(G)$ be a $k$-involution, $\theta \in \operatorname{Aut}(G)$ a Cartan involution satisfying $\sigma \theta=\theta \sigma, A$ a maximal $(\sigma, k)$-split torus of $G$ and $A^{0} \supset A$ a $\sigma$-stable maximal $k$-split torus of $G$. Then we have the following.

(i) $A^{0}$ is $H_{k}$-conjugate to a $\theta$-stable maximal $k$-split torus of $G$, which is also maximal $\theta$-split.

(ii) All $\sigma$-and $\theta$-stable maximal $k$-split tori of $G$ containing a maximal $(\sigma, k)$-split torus of $G$ are conjugate under $(H \cap K)_{k}^{0}$.

Proof. This result follows immediate from [23, 11.4] and [23, 11.18].

To show that $\sigma \theta$-stable parabolic $k$-subgroups are in fact $\sigma$-split we need the following:

Lemma 4.8. Let $P$ be a $\sigma \theta$-stable parabolic $k$-subgroup of $G$. Then $P \cap G_{\sigma \theta}$ is a parabolic $k$-subgroup of $G_{\sigma \theta}$. Moreover if $P$ is a minimal $\sigma \theta$-stable parabolic $k$-subgroup of $G$, then $P \cap G_{\sigma \theta}$ is a minimal parabolic $k$-subgroup of $G_{\sigma \theta}$.

Proof. Since $P$ is $\sigma \theta$-stable it follows from [23, Lemma 1.7] that $P G_{\sigma \theta}^{0}$ is closed in $G$. Let $B$ be a Borel subgroup of $P$ and consider the action of $B$ on $P H / H$. Since $P G_{\sigma \theta}^{0}$ is closed in $G, B$ has a closed orbit in $P H / H$. It follows that there is $x \in P$ such that $B x H$ is closed in $G$. By [35, Cor. 6.6 (i)], $x^{-1} B x$ is $\sigma \theta$-stable, and by $[31,5.1] x^{-1} B x \cap G_{\sigma \theta}^{0}$ is a Borel subgroup of $G_{\sigma \theta}^{0}$. Since $P \cap G_{\sigma \theta}^{0} \supset x^{-1} B x \cap G_{\sigma \theta}^{0}$, it follows that $P \cap G_{\sigma \theta}$ is a parabolic $k$-subgroup of $G_{\sigma \theta}$.

Assume next that $P$ is a minimal $\sigma \theta$-stable parabolic $k$-subgroup of $G$. Then by [23, Proposition 3.5] there exists a $\sigma \theta$-stable maximal $k$-split torus $A$ of $P$ such that $A_{\sigma \theta}^{+}$is a maximal $k$-split torus of $G_{\sigma \theta}$ and $Z_{G}\left(A_{\sigma \theta}^{+}\right)$is a $\sigma \theta$-stable Levi $k$-subgroup 
of $P$. But then $Z_{G}\left(A_{\sigma \theta}^{+}\right) \cap G_{\sigma \theta}$ is the Levi $k$-subgroup of a minimal parabolic $k$ subgroup of $G_{\sigma \theta}$, and consequently $P \cap G_{\sigma \theta}$ is a minimal parabolic $k$-subgroup of $G_{\sigma \theta}$.

Lemma 4.9. Let $\sigma$ and $\theta$ be as above. Then we have the following:

(i) $\theta\left|G_{\sigma \theta}=\sigma\right| G_{\sigma \theta}$ is a Cartan involution of $G_{\sigma \theta}$.

(ii) All $\theta$-stable maximal $k$-split tori of $G_{\sigma \theta}$ are maximal $(\sigma, k)$-split tori of $G$.

(iii) Every maximal $k$-split torus of $G_{\sigma \theta}$ is conjugate under $G_{\sigma \theta}^{0}(k)$ to a $\theta$-stable maximal $(\sigma, k)$-split torus of $G$.

Proof. (i) is immediate from $[23,11.9]$.

As for $(i i)$, assume that $A_{1}$ is a $\theta$-stable maximal $k$-split torus of $G_{\sigma \theta}$. Since $\theta\left|G_{\sigma \theta}=\sigma\right| G_{\sigma \theta}$ is a Cartan involution, it follows from [23, 11.4] that $A_{1}$ is a maximal $\theta$-split torus of $G_{\sigma \theta}$. Similarly $A_{1}$ is a maximal $\sigma$-split torus of $G_{\sigma \theta}$. That $A_{1}$ is a maximal $(\sigma, k)$-split torus of $G$ follows from the observation that $G_{\sigma \theta}$ contains a maximal $(\sigma, k)$-split torus of $G$. Namely, if $A_{2}$ is a $\theta$-stable maximal $k$-split torus with $\left(A_{2}\right)_{\sigma}^{-}$a maximal $(\sigma, k)$-split, then by $[23,11.4] A_{2}$ is also $\theta$-split and $\left(A_{2}\right)_{\sigma}^{-} \subset G_{\sigma \theta}$.

Finally, (iii) follows from (ii) and the fact that every maximal $k$-split torus of $G_{\sigma \theta}$ is conjugate under $G_{\sigma \theta}(k)$ to a $\theta$-stable maximal $k$-split torus. This proves the result.

The following result characterizes the facets corresponding to the $\sigma \theta$-stable parabolic $k$-subgroups of $G$.

Lemma 4.10. Let $P$ be a $\sigma \theta$-stable parabolic $k$-subgroup of $G, M$ a $\sigma \theta$-stable Levi $k$-subgroup of $P$ and $A$ a $\sigma \theta$-stable maximal $k$-split torus of $M$. Then there is a $\lambda \in X_{*}\left(A_{\sigma \theta}^{+}\right)$such that $P=P(\lambda)$ and $M=Z_{G}(\lambda)$.

Proof. Let $F$ be the facet with $P=P(F)$. Since $P(F)=\sigma \theta(P(F))=P(\sigma \theta(F))$, it follows that $\sigma \theta(F)=F$. Take $\delta \in X_{*}(A) \cap F$. Since $\sigma \theta(F)=F$, it follows that $\sigma \theta(\delta) \in F$ and $\lambda=\delta+\sigma \theta(\delta) \in X_{*}\left(A_{\sigma \theta}^{+}\right) \cap F$. Then $\lambda$ satisfies the above conditions.

Proposition 4.11. Every $\sigma \theta$-stable parabolic $k$-subgroup of $G$ is $\sigma$-split.

Proof. Let $P_{1}$ be a $\sigma \theta$-stable parabolic $k$-subgroup of $G$ and $P \subset P_{1}$ be a minimal $\sigma \theta$-stable parabolic $k$-subgroup of $G$. By Lemma $4.8, P \cap G_{\sigma \theta}$ is a minimal parabolic $k$-subgroup of $G_{\sigma \theta}$. Let $A$ be a $\theta$-stable maximal $k$-split torus of $G_{\sigma \theta}$ contained in $P$. It follows from Lemma 4.9 that $A$ is also a maximal $(\sigma, k)$-split torus of $G$. Let $A_{1} \supset A$ be a $\sigma \theta$-stable maximal $k$-split torus of $P$. From Lemma 4.10 it follows that there are $\lambda, \lambda_{1} \in X_{*}\left(\left(A_{1}\right)_{\sigma \theta}^{+}\right)$such that $P=P(\lambda)$ and $P_{1}=P\left(\lambda_{1}\right)$. Since $A$ is a maximal $k$ split torus of $G_{\sigma \theta}$, we have $\left(A_{1}\right)_{\sigma \theta}^{+}=A$. But then, since $A$ is $(\sigma, k)$-split, we have $\sigma(\lambda)=-\lambda$ and $\sigma\left(\lambda_{1}\right)=-\lambda_{1}$, and hence $P$ and $P_{1}$ are $\sigma$-split.

The converse of this result is not true. However, if a $\sigma$-split parabolic $k$-subgroup of $G$ contains a $\theta$-stable maximal $(\sigma, k)$-split torus of $G$, we have the following.

Proposition 4.12. Let $A$ be a $\theta$-stable maximal $(\sigma, k)$-split torus of $G, A^{0} \supset A$ a $\theta$-stable maximal $k$-split torus and $P \supset A$ a $\sigma$-split parabolic $k$-subgroup of $G$. Then we have the following:

(i) $A^{0}$ is maximal $\theta$-split.

(ii) $P$ is $\theta$-split. 
(iii) $P$ is $\sigma \theta$-stable.

(iv) $P$ is minimal $\sigma \theta$-stable if and only if $P$ is minimal $\sigma$-split.

Proof. ( $i$ ) follows from [23, 11.4], and (ii) follows from $(i)$ and Lemma 1.12.

As for $(i i)$, note that by Lemma 1.12 there exists $\lambda \in X_{*}(A)$ such that $P=P(\lambda)$. Since $A \subset A^{0}$ is $\theta$-split it follows that $\theta(\lambda)=-\lambda$, and therefore $P$ is $\theta$-split.

(iii) follows from (ii), since $P$ is both $\theta$-split and $\sigma$-split, and therefore

$$
\sigma \theta(P)=\sigma \theta(P(\lambda))=P(\sigma \theta(\lambda))=P(\lambda)=P .
$$

(iv). Assume first that $P \supset A$ is minimal $\sigma$-split. If $P_{1} \subset P$ is minimal $\sigma \theta$-stable, then by Proposition 4.11 $P_{1}$ is $\sigma$-split, and since $P$ is minimal $\sigma$-split it follows that $P_{1}=P$.

Conversely, assume $P \supset A$ is minimal $\sigma \theta$-stable. By Proposition $4.11 P$ is also $\sigma$-split. If $P_{1} \subset P$ is a minimal $\sigma$-split parabolic $k$-subgroup of $G$, then there exists a $\sigma$-stable maximal $k$-split torus $A_{1} \subset P_{1}$ with $A_{1}^{-}$a maximal $(\sigma, k)$-split torus of $G$. Similarly to $[23,2.4]$ it follows now that $A^{0}$ and $A_{1}$ are conjugate under $h \in\left(H \cap R_{u}(P)\right)_{k}$. So we may assume that $A_{1}=A^{0}$. But, then, since $P_{1}$ is $\sigma$-split, it follows from Lemma 1.12 that there exists $\lambda \in X_{*}\left(A_{1}^{-}\right)$such that $P_{1}=P(\lambda)$. The result now follows from Lemma 4.10.

Although not every $\sigma$-split parabolic $k$-subgroup of $G$ is $\sigma \theta$-stable, they are conjugate under $H_{k}$ with one which is $\sigma \theta$-stable.

Proposition 4.13. Every $\sigma$-split parabolic k-subgroup of $G$ is conjugate under $H_{k}$ with a $\sigma \theta$-stable parabolic $k$-subgroup.

Proof. Let $P_{1}$ be a $\sigma$-split parabolic $k$-subgroup of $G, P \subset P_{1}$ a minimal $\sigma$-split parabolic $k$-subgroup of $G$ and $A$ a maximal $(\sigma, k)$-split torus of $P$. From Proposition 4.7 it follows that there is an $h \in H_{k}$ such that $A_{1}=h A h^{-1}$ is $\theta$-stable. But then it follows from Proposition 4.12 that $A_{1}$ is $\theta$-split and $h P h^{-1}$ is $\sigma \theta$-stable. Similarly also $P_{1}$ is $\sigma \theta$-stable.

The above results give an adequate characterization of the $\sigma \theta$-stable parabolic $k$-subgroups. What is left is to show that we can restrict to $H_{k} \cap K_{k}$-conjugacy classes of $\sigma \theta$-stable parabolic $k$-subgroups instead of $H_{k}$-conjugacy classes. For this we first show the following:

Lemma 4.14. Let $A$ be a $\theta$-stable maximal $(\sigma, k)$-split torus of $G$. Then

$$
W_{G_{k}}(A) / W_{H_{k}}(A) \simeq W_{K_{k}}(A) / W_{\left(H_{k} \cap K_{k}\right)^{0}}(A) .
$$

Proof. Let $A^{0} \supset A$ be a $\theta$-stable maximal $k$-split torus. By Lemma $4.9 A^{0}$ is also $\theta$-split. Similarly to Lemma 3.2 , we can write

$$
W(A) \simeq W_{G_{k}}\left(A, A^{0}\right) / W_{0}(\sigma),
$$

where $W_{G_{k}}\left(A, A^{0}\right)=W(A) \cap W\left(A^{0}\right)=\left\{w \in W\left(A^{0}\right) \mid w(A) \subset A\right\}$ and $W_{0}(\sigma)$ is as in 1.7. The group $W_{G_{k}}\left(A, A^{0}\right)$ has representatives in $N_{G_{k}}\left(A_{k}, A_{k}^{0}\right)=\{n \in$ $\left.N_{G_{k}}\left(A_{k}^{0}\right) \mid n A n^{-1} \subset A\right\}$. By $[23,11.5] N_{G_{k}}\left(A_{k}^{0}\right)=A_{k}^{0} \cdot N_{K_{k}}\left(A_{k}^{0}\right)$, so it follows that also

$$
N_{G_{k}}\left(A_{k}, A_{k}^{0}\right)=A_{k}^{0} \cdot N_{K_{k}}\left(A_{k}, A_{k}^{0}\right) .
$$

The root system $\Phi_{0}(\sigma)$ can be identified with the root system $\Phi\left(A_{1}, G_{1}\right)$, where $G_{1}=D\left(Z_{G}(A)\right)=\left[Z_{G}(A), Z_{G}(A)\right]$ and $A_{1}=\left(A^{0} \cap G_{1}\right)^{0}$. By $[23,11.9] G_{1}$ is also a group with a Cartan involution. Since $A$ is a maximal $(\sigma, k)$-split torus of $G$, the 
torus $A_{1}$ is a maximal $\theta$-split torus of $G$, contained in $H \cap G_{1}$. It follows from [23, 11.5] that $W_{0}(\sigma)=W\left(A_{1}, G_{1}\right)$ has representatives in $\left(H \cap K \cap G_{1}\right)(k)$. Combining this with (4.2), we get

$$
W_{G_{k}}\left(A, A^{0}\right) / W_{0}(\sigma) \simeq W_{K_{k}}\left(A, A^{0}\right) / W_{0}(\sigma) .
$$

Combined with (4.1), this gives us

$$
W_{G_{k}}(A) \simeq W_{K_{k}}(A) .
$$

Since all $\sigma$-stable maximal $k$-split tori of $Z_{G}(A)$ are conjugate under $(H \cap K \cap$ $\left.Z_{G}(A)\right)^{0}$ and since $W_{0}(\sigma)$ has representatives in $\left(H \cap K \cap Z_{G}(A)\right)(k)$, we also have the following identifications:

$$
W_{H_{k}}(A) \simeq W_{H_{k}}\left(A, A^{0}\right) / W_{0}(\sigma)
$$

and

$$
W_{(K \cap H)_{k}^{0}}(A) \simeq W_{(K \cap H)_{k}^{0}}\left(A, A^{0}\right) / W_{0}(\sigma) .
$$

So it suffices to show that $W_{H_{k}}\left(A, A^{0}\right) \simeq W_{(K \cap H)_{k}}\left(A, A^{0}\right)$. So let $h \in H_{k}$ be a representative of $w \in W_{H_{k}}\left(A, A^{0}\right)$. By (4.2) we can write

$$
h=a_{1} a_{2} p k_{1} \text { with } a_{1} \in\left(A^{0}\right)_{\sigma}^{+}=, a_{2} \in\left(A^{0}\right)_{\sigma}^{-}=A, p \in \tau(K), k_{1} \in(K \cap H)_{k}^{0} .
$$

Since $\sigma(h)=h$ it follows that $a_{2}^{2}=p^{-2}$, and hence $a_{2}^{4}=p^{-4}=$ id. On the other hand, since $a_{2} \in A_{k}, p \in K_{k}$ and since $-1 \notin\left(k^{\times}\right)^{2}=\left(k^{\times}\right)^{4}$ it follows that we must have $a_{2}^{2}=p^{-2}=$ id. So $p \in(K \cap H)_{k}$ and $a=a_{1} a_{2} \in\left(A^{0} \cap H\right)_{k}$. It follows that

$$
W_{H_{k}}\left(A, A^{0}\right) \simeq W_{(K \cap H)_{k}}\left(A, A^{0}\right),
$$

which proves the result.

Proposition 4.15. Let $P_{1}$ and $P_{2}$ be $\sigma \theta$-stable parabolic $k$-subgroups of $G$. Then $P_{1}$ and $P_{2}$ are conjugate under $H_{k}$ if and only if they are conjugate under $H_{k} \cap K_{k}$.

Proof. Let $h \in H_{k}$ be such that $h P_{1} h^{-1}=P_{2}$. Assume first that $P_{1}$ and $P_{2}$ are minimal $\sigma \theta$-stable parabolic $k$-subgroups of $G$. Let $A_{1} \subset P_{1}$ and $A_{2} \subset P_{2}$ be $\theta$-stable maximal $(\sigma, k)$-split tori of $G$. Then $h A_{1} h^{-1} \subset P_{2}$ is a $(\sigma, k)$-split torus of $G$. Since $h A_{1} h^{-1}$ is $\sigma$-stable, we get $h A_{1} h^{-1} \subset P_{2} \cap \sigma\left(P_{2}\right)=Z_{G}\left(A_{2}\right)$. So $h A_{1} h^{-1} A_{2} \subset Z_{G}\left(A_{2}\right)$ is a $(\sigma, k)$-split torus of $G$. From the maximality of $A_{2}$ it follows that $h A_{1} h^{-1}=A_{2}$. On the other hand it follows from Proposition 4.7 that there exists $h_{1} \in(H \cap K)_{k}^{0}$ such that $h_{1} A_{1} h_{1}^{-1}=A_{2}$. Let $P=h_{1} P_{1} h_{1}^{-1}$ and $n=h h_{1}^{-1} \in N_{H_{k}}\left(A_{2}\right)$. Then both $P$ and $P_{2}$ are contained in $\mathfrak{P}\left(A_{2}\right)$, and $n \mathrm{Pn}^{-1}=P_{2}$. It follows now from Lemma 4.14 that $P$ and $P_{2}$ are conjugate under $N_{(H \cap K)_{k}^{0}}\left(A_{2}\right)$, which proves the result for $P_{1}$ and $P_{2}$ minimal $\sigma \theta$-stable parabolic $k$-subgroups of $G$.

If $P_{1}$ and $P_{2}$ are not minimal, then let $P \subset P_{1}$ be a minimal $\sigma \theta$-stable parabolic $k$-subgroup of $G$ and $A \subset P$ a $\theta$-stable maximal $(\sigma, k)$-split torus of $G$. Then $h P h^{-1} \subset P_{2}$ is a minimal $\sigma$-split parabolic $k$-subgroup of $G$. Let $\tilde{P} \subset P_{2}$ be a minimal $\sigma \theta$-stable parabolic $k$-subgroup of $G$ and $\tilde{A} \subset \tilde{P}$ a $\theta$-stable maximal $(\sigma, k)$ split torus of $G$. Since $h A h^{-1}$ and $\tilde{A} \subset P_{2}$ are maximal $(\sigma, k)$-split, there exists $h_{1} \in\left(P_{2} \cap H\right)_{k}$ such that $h_{1} h A_{1} h^{-1} h_{1}^{-1}=\tilde{A}$. Now $P_{0}=h_{1} h P h^{-1} h_{1}^{-1} \subset P_{2}$ is minimal $\sigma$-split, and $\tilde{A} \subset P_{0}$. By Lemma 1.12 there exists $\lambda \in X_{*}(\tilde{A})$ such that $P_{0}=P(\lambda)$. Since by Proposition $4.7 \tilde{A}$ is $\theta$-split, it follows that $\theta(\lambda)=-\lambda$ and hence $P_{0}$ is $\theta$-split. Since $P_{0}$ is also $\sigma$-split, it follows that $P_{0}$ is $\sigma \theta$-stable. From 
the first part of this proof it follows now that there exists $h_{0} \in(H \cap K)_{k}^{0}$ such that $\tilde{P}=h_{0} P h_{0}^{-1}$. Let $\tilde{P}_{1}=h_{0} P_{1} h_{0}^{-1}$. Then $\tilde{P}_{1}$ and $P_{2}$ are conjugate under $n=h_{1} h h_{0}^{-1} \in N_{H_{k}}\left(A_{0}\right)$. From Lemma 4.14 it follows now that $\tilde{P}_{1}$ and $P_{2}$ are conjugate under $N_{(H \cap K)_{k}^{0}}\left(A_{2}\right)$, and consequently $P_{1}$ and $P_{2}$ are conjugate under $(H \cap K)_{k}^{0}$.

Combining the above results with those of section 2, we now obtain the following result:

Theorem 4.16. Let $A$ be a $\theta$-stable maximal $(\sigma, k)$-split torus of $G, A^{0} \supset A$ a $\theta$-stable maximal $k$-split torus of $G, \Delta$ a $\sigma$-basis of $\Phi\left(A^{0}\right)$ and $\Delta_{0}(\sigma)=\{\alpha \in \Delta \mid$ $\sigma(\alpha)=\alpha\}$. Then we have the following.

(i) There is a bijective correspondence between the $H_{k}$-conjugacy classes of the minimal $\sigma$-split parabolic k-subgroups in $\mathfrak{P}(A)$ and the $H_{k} \cap K_{k}$-conjugacy classes of the $\sigma \theta$-stable parabolic $k$-subgroups in $\mathfrak{P}(A)$.

(ii) If $P$ is any minimal $\sigma \theta$-stable parabolic $k$-subgroup of $G$, then there exist $h \in(H \cap K)_{k}^{0}$ and $n \in N_{K_{k}}\left(A^{0}\right) \cap N_{K_{k}}(A)$ such that $n h P h^{-1} n^{-1}=P_{\Delta_{0}(\sigma)}$.

Proof. ( $i$ ) is immediate from Proposition 4.15. As for (ii), note first that by [23, 11.4] $N_{G_{k}}\left(A_{k}^{0}\right)=A_{k}^{0} \cdot N_{K_{k}^{0}}\left(A_{k}^{0}\right)$. Since $W(A) \simeq W\left(A, A^{0}\right) / W_{0}(\sigma)$, it follows from Lemma 1.10 that $W(A)$ has representatives in $N_{K_{k}^{0}}\left(A_{k}^{0}\right)$. Now the result follows from Theorem 2.9 .

Similarly we have the following generalization of Theorem 3.1.

Theorem 4.17. Let $A$ be a $\theta$-stable maximal $(\sigma, k)$-split torus of $G$ and $P \supset A$ a minimal $\sigma \theta$-stable parabolic $k$-subgroup of $G$. There is a one to one correspondence between the $H_{k} \times P_{k}$-orbits on $G_{k}$ contained in $(H P)_{k}$ and $W_{K_{k}}(A) / W_{\left(H_{k} \cap K_{k}\right)^{0}}(A)$. In particular the orbits are given by $H_{k} n P_{k}$ with $n \in N_{K_{k}}(A)$ a representative for the coset $w$ in $W_{K_{k}}(A) / W_{\left(H_{k} \cap K_{k}\right)^{0}}(A)$.

Proof. Note first that by Proposition $4.12(i v) P$ is also minimal $\sigma$-split. Since by Proposition 4.7 all maximal $(\sigma, k)$-split tori are conjugate under $H_{k}$, the result follows from Lemma 4.14 and Theorem 3.1.

We conclude this section with a generalization of Proposition 3.3 to the setting of groups with a Cartan involution.

Proposition 4.18. Let $A$ be a $\theta$-stable maximal $(\sigma, k)$-split torus of $G, P \supset A$ a minimal $\sigma \theta$-stable parabolic $k$-subgroup of $G$ and $A^{0} \supset A$ a $\theta$-stable maximal $k$-split torus of $G$. If $H_{k} g P_{k}$ is an $H_{k} \times P_{k}$-orbit on $G_{k}$ which is not contained in $(H P)_{k}$, then there exists $x \in K_{k}$ such that $H_{k} x P_{k}=H_{k} g P_{k}, x A^{0} x^{-1}$ is both $\theta$-and $\sigma$-stable and $\operatorname{dim}\left(x A^{0} x^{-1} \cap H\right)>\operatorname{dim}\left(\left(A^{0}\right)^{+}\right)$.

Proof. Let $H_{k} g P_{k}$ be an $H_{k} \times P_{k}$-orbit on $G_{k}$ which is not contained in $(H P)_{k}$. By Proposition 3.3 there exists $x \in G_{k}$ such that $H_{k} x P_{k}=H_{k} g P_{k}, x A^{0} x^{-1}$ is $\sigma$ stable and $\operatorname{dim}\left(x A^{0} x^{-1} \cap H\right)>\operatorname{dim}\left(\left(A^{0}\right)^{+}\right)$. We need to show that we can actually choose $x$ in $K_{k}$. By Proposition 4.7 there exists $h \in H_{k}$ such that $h x A^{0} x^{-1} h^{-1}$ is maximal $\theta$-split. From $[23,11.4]$ it follows that there exists $k_{1} \in K_{k}^{0}$ such that $k_{1} A^{0} k_{1}^{-1}=h x A^{0} x^{-1} h^{-1}$. Now $H_{k} P_{k}$ and $H_{k} k_{1}^{-1} x P_{k}$ are orbits containing $A$, so there exists $w \in W\left(A, A^{0}\right)$ such that $H_{k} w k_{1}^{-1} x P_{k}=H_{k} P_{k}$. By Lemma 4.14 $w$ has a representative $k_{2} \in N_{K_{k}}\left(A^{0}, A\right)$. From this it follows that $H_{k} x P_{k}=$ 
$H_{k} k_{1} k_{2}^{-1} P_{k}$, and since clearly $k_{1} k_{2}^{-1} A^{0} k_{2} k_{1}^{-1}=h x A^{0} x^{-1} h^{-1}$ is $\theta$ - and $\sigma$-stable and $\operatorname{dim}\left(k_{1} k_{2}^{-1} A^{0} k_{2} k_{1}^{-1} \cap H\right)>\operatorname{dim}\left(\left(A^{0}\right)^{+}\right)$, the result follows.

\section{REFERENCES}

1. E. van den Ban, The principal series for a reductive symmetric space $I$. $H$-fixed distribution vectors, Ann. Sci. Ec. Norm. Sup. 21 (1988), 359-412. MR 90a:22016

2. van den Ban, E. and Schlichtkrull, H., The most continuous part of the Plancherel decomposition for a reductive symmetric space, Ann. of Math. (2) 145 (1997), 267-364. CMP 97:10

3. E. Becker, Valuations and real places in the theory of formally real fields, Géométrie Algébrique Réelle et Formes Quadratiques (Berlin-Heidelberg-New York) (J.-L. ColliotThélène, M. Coste, L. Mahé, et M.-F. Roy, ed.), Lecture Notes Mathematics, vol. 959, Springer Verlag, 1982, pp. 1-40. MR 84f:12011

4. M. Berger, Les espaces symétriques non-compacts, Ann. Sci. École Norm. Sup. 4 (1957), 85-177. MR 21:3516

5. A. Borel and J. Tits, Groupes réductifs, Inst. Hautes Études Sci. Publ. Math. 27 (1965), 55-152. MR 34:7527

6. , Compléments a l'article "groupes réductifs", Inst. Hautes Études Sci. Publ. Math. 41 (1972), 253-276. MR 47:3556

7. F. Bruhat and J. Tits, Groupes réductifs sur un corps local, Inst. Hautes Études Sci. Publ. Math. 41 (1972), 5-252. MR 48:6265

8. J.-L. Brylinski and P. Delorme, Vecteurs distributions $H$-invariants pour les séries principales géneralisées d'espaces symétriques réductifs et prolongement méromorphe d'integrales d'Eisenstein, Invent. Math. 109 (1992), 619-664. MR 93m:22016

9. M. Flensted-Jensen, Discrete series for semisimple symmetric spaces, Annals of Math. 111 (1980), 253-311. MR 81h:22015

10. Harish-Chandra, Harmonic analysis on real reductive groups I. The theory of the constant term, J. Funct. Anal. 19 (1975), 103-204. MR 53:3201

11. Harmonic analysis on real reductive groups II. Wave packets in the Schwartz space, Invent. Math. 36 (1979), 1-55. MR 55:12874

12. - Harmonic analysis on real reductive groups III. The Maass-Selberg relations and the Plancherel formula, Annals of Math. 104 (1976), 117-201. MR 55:12875

13. _ Harish-Chandra collected papers, Springer-Verlag, New York, 1984. MR 85e:01061

14. S. Helgason, Differential geometry, Lie groups and symmetric spaces, Pure and Applied Mathematics, vol. XII, Academic Press, New York, 1978. MR 80k:53081

15. A. G. Helminck, On the classification of symmetric $k$-varieties I, To appear.

16. _ Algebraic groups with a commuting pair of involutions and semisimple symmetric spaces, Adv. in Math. 71 (1988), 21-91. MR 90a:17011

17. MR 92a:20047

18. 1-92. CMP 98:02

19. _ Tori invariant under an involutorial automorphism III, To appear.

20. On groups with a Cartan involution, Proceedings of the Hyderabad Conference on Algebraic Groups (Hyderabad, India), National Board for Higher Mathematics, 1992, pp. 151192. MR 92j:20042

21. _ Symmetric k-varieties, Algebraic Groups and Their Generalizations: Classical Methods (Providence, RI), vol. 56, Proc. Sympos. Pure Math., no. Part 1, Amer.Math. Soc, 1994, pp. 233-279. MR 92j:20042

22. A. G. Helminck and G. F. Helminck, $H_{k}$-fixed distributionvectors for representations related to $\mathfrak{p}$-adic symmetric varieties, To appear.

23. A. G. Helminck and S. P. Wang, On rationality properties of involutions of reductive groups, Adv. in Math. 99 (1993), 26-96. MR 94d:20051

24. J. E. Humphreys, Linear algebraic groups, Springer-Verlag, Berlin, 1975. MR 53:633

25. H. Jacquet, K. Lai, and S. Rallis, A trace formula for symmetric spaces, Duke Math. J. 70 (1993). MR 94d:11033

26. G. Lusztig, Symmetric spaces over a finite field, The Grothendieck Festschrift Vol. III (Boston, MA), Progr. Math., vol. 88, Birkhäuser, 1990, pp. 57-81. MR 92e:20034 
27. T. Matsuki, The orbits of affine symmetric spaces under the action of minimal parabolic subgroups, J. Math. Soc. Japan 31 (1979), 331-357. MR 81a:53049

28. T. Oshima and T. Matsuki, A description of discrete series for semisimple symmetric spaces, Adv. Stud. in Pure Math., vol. 4, Academic Press, Orlando, FL, 1984, pp. 331-390. MR 87m:22042

29. T. Oshima and J. Sekiguchi, Eigenspaces of invariant differential operators in an affine symmetric space, Invent. Math. 57 (1980), 1-81. MR 81k:43014

30. A. Prestel, Lectures on formally real fields, Lecture Notes Mathematics, vol. 1093, Springer Verlag, Berlin-Heidelberg-New York, 1984. MR 86h:12013

31. R.W. Richardson, Orbits, invariants and representations associated to involutions of reductive groups, Invent. Math. 66 (1982), 287-312. MR 83i:14042

32. W. Rossmann, The structure of semisimple symmetric spaces, Canad. J. Math. 31 (1979), 157-180. MR 81i:53042

33. I. Satake, Classification theory of semisimple algebraic groups, Lecture Notes in Pure and Appl. Math., vol. 3, Dekker, Berlin, 1971. MR 47:5135

34. T. A. Springer, Linear algebraic groups, Progr. Math., vol. 9, Birkhäuser, Boston-BaselStuttgart, 1981. MR 84i:20002

35. S_ Some results on algebraic groups with involutions, Algebraic groups and related topics, Adv. Stud. in Pure Math., vol. 6, Academic Press, Orlando, FL, 1984, pp. 525-543. MR 86m:20050

36. J. A. Wolf, Finiteness of orbit structure for real flag manifolds, Geom. Dedicata 3 (1974), 377-384. MR 51:943

Department of Mathematics, North Carolina State University, Raleigh, North CarOLINA, 27695-8205

E-mail address: loek@math.ncsu.edu

Department of Mathematics, Universiteit Twente, Enschede, The Netherlands

E-mail address: helminck@math.utwente.nl 